\title{
Loss of transverse Landau damping by noise and wakefield driven diffusion
}

\author{
Sondre Vik Furuseth ${ }^{*}$ \\ CERN, 1211 Geneva, Switzerland, and EPFL, 1015 Lausanne, Switzerland \\ Xavier Buffat \\ CERN, 1211 Geneva, Switzerland
}

(Received 14 July 2020; accepted 2 November 2020; published 30 November 2020)

\begin{abstract}
Landau damping of coherent modes is strongly dependent on the exact shape of the particle bunches. One often assumes that the transverse distributions in high-energy hadron colliders can be approximated by Gaussian distributions, in acceptable agreement with measurements, but known to be only a first approximation. In this paper, it is investigated how a specific change of the transverse distributions can cause a loss of Landau damping. A mechanism is introduced where the coherent modes, which are excited by noise in the machine, act back on the individual particles through wakefields. The impact is modeled as a narrow diffusion in frequency space, and therefore also in action space due to amplitude dependent detuning, which leads to a local flattening of the distribution. This distribution evolution corresponds to the drilling of a borehole in the stability diagram, i.e. a local reduction of the imaginary part of the curve. Hence, initially stable regions are changed into unstable ones at the real frequencies of the coherent modes. To mitigate this instability mechanism, one must operate the machine with a stability margin of magnitude that depends on the noise amplitude and the coherent modes. In this model, the latency is defined as the time from the start of the noise excitation, on an initially Gaussian distributed bunch, to the bunch instability. The proposed model is found to agree with results in dedicated latency experiments performed in the LHC, where bunches eventually went unstable with more than twice the detuning strength required for the stabilization of a Gaussian distribution.
\end{abstract}

DOI: $10.1103 /$ PhysRevAccelBeams.23.114401

\section{INTRODUCTION}

Circular high-energy hadron colliders, such as the Large Hadron Collider (LHC), depend on Landau damping for the stabilization of the beams. Landau damping prevents the self-amplification of coherent modes. The amplitudes of the discrete modes evolve like $\exp \left(-i 2 \pi Q_{\text {coh }} T\right)$, where $Q_{\text {coh }}$ is the complex coherent tune and $T$ is the turn number. Hence, if $\operatorname{Im}\left\{Q_{\text {coh }}\right\}>0$, the mode will grow exponentially. A necessary requirement for Landau damping is that the individual particles must have a spectrum of particle tunes $Q$ that contain the tune of the unstable mode. In the weak head-tail approximation the effect of Landau damping can be considered by the stability diagram [1]

$$
\frac{-1}{\Delta Q_{\mathrm{coh}, j}}=\int_{0}^{\infty} \mathrm{d} J_{x} \int_{0}^{\infty} \mathrm{d} J_{y} \frac{J_{j} \frac{\mathrm{d} \Psi_{\mathrm{eq}}\left(J_{x}, J_{y}\right)}{\mathrm{d} J_{j}}}{Q-Q_{j}\left(J_{x}, J_{y}\right)+i \epsilon},
$$

\footnotetext{
*sondre.vik.furuseth@cern.ch
}

Published by the American Physical Society under the terms of the Creative Commons Attribution 4.0 International license. Further distribution of this work must maintain attribution to the author(s) and the published article's title, journal citation, and DOI. where $\Delta Q_{\text {coh }, j}$ would be the complex coherent tune shift in the transverse plane $j$, if there was no tune spread $Q_{j}\left(J_{x}, J_{y}\right)$. Due to the tune spread, the coherent tune changes to $Q+i \epsilon \equiv Q_{\mathrm{LD}}, Q \in \mathbb{R}$. In the limit $\epsilon \rightarrow 0$, the left-hand side gives the modified limit of stability on the coherent tune shift. Note that the tunes $Q$ and the related (angular) frequencies $\omega \equiv 2 \pi f_{\text {rev }} Q \equiv \omega_{\text {rev }} Q$ will be referred to interchangeably throughout this paper.

Instabilities were observed in the LHC in conditions where Eq. (1) predicts stability with a margin of approximately a factor 2 [2]. The uncertainty on the tune spread $[3,4]$ and the coherent tune shift [5] are not sufficient to explain this discrepancy. The cause may therefore be found in the description of the beam distribution. In addition, the observations of instabilities exhibited latencies ranging from a few to tens of minutes [2]. In an experiment described in Sec. V C [6,7], the role of an external source of noise in the latent instability mechanism was confirmed. Consequently, we seek to study and explain the possible mechanisms that systematically drive the evolution of the transverse distribution toward something non-Gaussian, and the corresponding possible loss of Landau damping [8,9].

Loss of Landau damping due to a change of the bunch distribution is assumed to primarily be driven by stochastic 
processes that can be modeled as a diffusion. The problematic diffusion cannot be uniform, but must be peaked at a critical frequency: the frequency of a mode that requires stabilization by Landau damping. The focus of this paper is a mechanism driven by decoherence, in which the energy from the coherent modes is transferred to the betatron motion of the resonant individual particles through wakefields. Other mechanisms can cause a similar frequency dependent diffusion, but will not systematically be peaked at a critical frequency. If the modes are stable, the energy they carry is typically assumed to be infinitesimal. However, since the beam is excited by external noise sources, e.g., the ground motion, the power converter ripples, or the transverse feedback system, the modes will be excited to finite amplitudes and carry a non-negligible energy. By transferring this energy to the resonant particles, the reserve of Landau damping will be depleted over time.

\section{NOISE EXCITED WAKEFIELDS}

In a collision-free Hamiltonian system, Liouville's theorem states that the distribution function $\Psi$ is constant along any trajectory of the system in phase space [10]. For non-colliding particles under the influence of the Hamiltonian $\mathcal{H}$, the Liouville theorem can be stated mathematically by the Vlasov equation [11-15]

$$
\frac{\mathrm{d} \Psi}{\mathrm{d} t}=\frac{\partial \Psi}{\partial t}+[\Psi, \mathcal{H}]=0
$$

where the square brackets are the Poisson brackets.

The impact of noise excited wakefields will be derived from the Vlasov equation with the perturbed one-turn Hamiltonian given by

$$
\mathcal{H}=\mathcal{H}_{0}+\epsilon \mathcal{H}_{1}=\mathcal{H}_{0}+\epsilon \mathcal{H}_{\text {wake }}+\epsilon \mathcal{H}_{\text {noise }},
$$

consisting of an equilibrium Hamiltonian $\mathcal{H}_{0}$, which governs the unperturbed motion over one turn around the synchrotron, and perturbations $\mathcal{H}_{\text {wake }}$ and $\mathcal{H}_{\text {noise }}$, which model the assumed weak wakefields and external noise, respectively. The artificial tag $\epsilon$ denotes the size of each term, $\mathcal{O}\left(\epsilon^{n}\right) \gg \mathcal{O}\left(\epsilon^{n+1}\right)$, which is useful in grouping terms of equal order. In the end, $\epsilon$ will be set to 1 . The perturbation representing the impact of the wakefields, $\mathcal{H}_{\text {wake }}$, models the collective wake force from the full ensemble of particles, as was first suggested by A. Vlasov [11]. The short-range interactions between individual particles are in this manner neglected, as required by the Vlasov equation. Such perturbations to the Hamiltonian can be constructed based on the dipolar wake potentials within the ultrarelativistic approximation, as have been done in [12-17]. In this paper, the perturbation $\mathcal{H}_{\text {wake }}$ is assumed to only represent the transverse kicks from weak dipolar wakefields.
The distribution can similarly be expanded as

$$
\Psi=\Psi_{0}+\epsilon \Psi_{1}+\epsilon^{2} \Psi_{2},
$$

consisting of a centered initial distribution $\Psi_{0}$, which drives no dipolar wakefields, a quickly oscillating perturbation $\Psi_{1}$, and a second order perturbation $\Psi_{2}$, which is assumed to not drive dipolar wakefields.

The Vlasov equation can be organized by order as

$$
\begin{gathered}
\frac{\partial \Psi_{0}}{\partial t}+\left[\Psi_{0}, \mathcal{H}_{0}\right]=0 \\
\frac{\partial \Psi_{1}}{\partial t}+\left[\Psi_{1}, \mathcal{H}_{0}\right]=-\left[\Psi_{0}, \mathcal{H}_{\text {wake }}\right]-\left[\Psi_{0}, \mathcal{H}_{\text {noise }}\right], \\
\frac{\partial \Psi_{2}}{\partial t}+\left[\Psi_{2}, \mathcal{H}_{0}\right]=-\left[\Psi_{1}, \mathcal{H}_{\text {wake }}\right]-\left[\Psi_{1}, \mathcal{H}_{\text {noise }}\right]
\end{gathered}
$$

The leading order distribution term $\Psi_{0}$ is not affected by the perturbations. The four driving terms on the right-hand side (rhs) must be considered in detail: (i) The first driving term on the rhs of Eq. (5b) is the excitation of the perturbation due to wakefields, often referred to as the linearized Vlasov equation. Assuming no tune spread or noise, the linearized Vlasov equation will be solved to get the wakefield driven eigenmodes with complex eigenfrequencies $\omega_{m}$ in Sec. II B. Due to the tune spread, the discrete mode mixes with the incoherent spectrum, and the complex eigenfrequencies are changed to $\Omega_{m}$. If $\operatorname{Im}\left\{\Omega_{m}\right\}>0$, the mode is already unstable. The interesting case in this paper is $\operatorname{Im}\left\{\Omega_{m}\right\}<0$, for which $\Omega_{m}$ will be found in Sec. II C; (ii) The second driving term on the rhs of Eq. (5b) models the excitation of the perturbation $\Psi_{1}$ by the noise. How much the external noise excites the modes will be discussed in Sec. II D. Due to the noise, the amplitudes of the perturbations will no longer be infinitesimal, even if the mode is stable, and one must consider the driving terms in Eq. (5c); (iii) The first term on the rhs of Eq. (5c) models the effect of the noise excited wakefields on the incoherent particles, found in Sec. II E to lead to a slow and narrow diffusion in frequency space. This is the main topic of this paper; (iv) The second term on the rhs of Eq. (5c) models the direct noise driven diffusion. This terms was studied in [9] and found to not be critical in loosing Landau damping. The combined effect is a slow, yet steady, evolution of the equilibrium distribution $\Psi_{\text {eq }}=\Psi_{0}+\epsilon^{2} \Psi_{2}$, which in critical configurations will lead to an instability by loss of Landau damping, after a latency that is estimated in Sec. II F.

\section{A. Single-particle dynamics}

The individual particles in a bunch will be described by their normalized canonical coordinates in either transverse plane [18] 


$$
\begin{aligned}
& y=\frac{Y}{\sqrt{\beta \varepsilon_{0}}}=\sqrt{2 J} \cos (\phi), \\
& p=\frac{1}{\sqrt{\beta \varepsilon_{0}}}\left(\alpha Y+\beta \frac{\mathrm{d} Y}{\mathrm{~d} s}\right)=-\sqrt{2 J} \sin (\phi),
\end{aligned}
$$

where $Y$ is the offset from the design orbit, $s$ is the longitudinal coordinate along the accelerator, $\alpha$ and $\beta$ are the Twiss parameters, $\varepsilon_{0}$ is the initial geometrical beam emittance, and $\phi$ is the canonical conjugate of $J$, which is the normalized absolute particle action, given in units of $\varepsilon_{0}$.

In the case when $\phi=\phi_{0}+\omega t$, where $\omega$ is the constant incoherent betatron frequency, and the particle receives impulses $\Delta p(t)$, the perturbed Hamiltonian is

$$
\mathcal{H}=\omega J-y \Delta p=\omega \frac{y^{2}+p^{2}}{2}-y \Delta p,
$$

such that Hamilton's equations give

$$
\ddot{y}+\omega^{2} y=\omega \Delta p .
$$

For Landau damping to exist, there must be a frequency spread within the bunch. Usually, this is achieved by making the frequency of single particles depend on their transverse actions using Landau octupoles [19]. Throughout this paper, a linear dependence of the transverse frequencies will be considered

$$
\begin{aligned}
& \omega_{x}\left(J_{x}, J_{y}\right)=\omega_{x 0}+\tilde{a}_{x} J_{x}+\tilde{b}_{x} J_{y}, \\
& \omega_{y}\left(J_{x}, J_{y}\right)=\omega_{y 0}+\tilde{b}_{y} J_{x}+\tilde{a}_{y} J_{y} .
\end{aligned}
$$

This is more commonly discussed as a tune spread

$$
\begin{aligned}
& Q_{x}\left(J_{x}, J_{y}\right)=Q_{x 0}+a_{x} J_{x}+b_{x} J_{y}, \\
& Q_{y}\left(J_{x}, J_{y}\right)=Q_{y 0}+b_{y} J_{x}+a_{y} J_{y} .
\end{aligned}
$$

Typically, the prefactors $a_{j}$ and $b_{j}$ are different for the different planes and beams. That is not relevant in this derivation, and the subscripts will be omitted from here on, implying $a_{x}=a_{y}=a$ and $b_{x}=b_{y}=b$.

The longitudinal coordinates of a particle, when above transition, will be given as [18]

$$
\begin{aligned}
z & =\sqrt{2 I_{z} \beta_{z}} \cos \left(\phi_{z 0}+\omega_{s} t\right), \\
\frac{\Delta p}{p_{0}} & =\delta=\sqrt{\frac{2 I_{z}}{\beta_{z}}} \sin \left(\phi_{z 0}+\omega_{s} t\right),
\end{aligned}
$$

where $z=s-s_{0}(t)$ is the longitudinal position relative to the synchronous particle, $I_{z}$ is the longitudinal nonnormalized action, and $\omega_{s}$ is the synchrotron frequency, which is assumed to be small.

If there is a nonzero linear chromaticity $Q^{\prime}=\omega^{\prime} / \omega_{\text {rev }}$, the transverse betatron frequencies will depend on the relative momentum mismatch as

$$
\omega_{0} \rightarrow \omega_{0}+\omega^{\prime} \delta
$$

Thus, the frequency of the betatron oscillation is oscillating as well. In the limit of small $\omega_{s}$, it can be shown that the single-particle motion, starting at $\phi_{0}=0$ and $\phi_{z 0}=-\pi / 2$ for ease of notation, can be described by a Fourier series

$$
\begin{aligned}
\cos [\phi(t)] & =\cos \left[\omega_{0} t+\int_{0}^{t} \omega^{\prime} \delta \mathrm{d} t\right] \\
& =\sum_{n=-\infty}^{\infty} J_{n}\left(\frac{\omega^{\prime} \sqrt{2 I_{z} / \beta_{z}}}{\omega_{s}}\right) \cos \left[\left(\omega_{0}+n \omega_{s}\right) t\right],
\end{aligned}
$$

where $J_{n}(\cdot)$ are the Bessel functions of the first kind, acting as coefficients of the various synchrotron sidebands.

\section{B. Wakefield eigenmodes $-\omega_{m}$}

It is common to assess beam stability by neglecting noise and assuming weak wakefield driven perturbations, in which case Eq. (5) becomes the linearized Vlasov equation. The dipolar wake force is only dependent on $z$, not $\delta$. It is common to model it as one effective kick $P_{\text {coh }}(z, t)$ per turn. One can solve the Vlasov equation with a normal mode analysis, assuming that the distribution perturbation in either transverse plane can be written as a sum of orthogonal modes [14,15]

$$
\begin{aligned}
\Psi_{1} & =\sum_{m} \Delta \Psi_{m}\left(J, \phi, I_{z}, \phi_{z}, t\right) \\
& =\sum_{m} f_{m}\left(J, \phi, I_{z}\right) e^{-i \phi_{m}\left(t, \phi_{z}, z\right)},
\end{aligned}
$$

with phases

$\phi_{m}\left(t, \phi_{z}, z\right)=\phi_{m 0}+\left(\omega_{m}+l_{m} \omega_{s}\right) t-l_{m} \phi_{z}-\frac{z \omega^{\prime}}{\omega_{s} \beta_{z}}$.

Here, $\omega_{m}=\omega_{0}+\Delta \omega_{m}$ is the complex frequency of mode $m \neq 0, \Delta \omega_{m}$ is the complex frequency shift generated by the wakefields, $l_{m}$ is the angular mode number, $\omega_{0}$ is the bare betatron frequency of the synchronous particle, and the last term is the headtail phase factor. Here, it has been assumed that a mode consists of a single angular spatial frequency. The mode number $m$ is an iterative index.

The individual particles are oscillating in longitudinal phase space, given by Eq. (11). The phase of the coherent mode at the location of an individual particle is

$$
\phi_{\Delta m}(t)=\left(\phi_{m 0}-l_{m} \phi_{z 0}\right)+\omega_{m} t-\frac{z(t) \omega^{\prime}}{\omega_{s} \beta_{z}},
$$

where the synchrotron frequency has been canceled. This phase evolves with time equally to the transverse phase of the single particle in Eq. (13), illustrating that the coherent 
mode in fact consists of the motion of synchronized individual particles.

The average transverse complex offset of the distribution in the $y$-plane, following the longitudinal motion of the single particles, can be written in terms of normalized, fixed eigenfunctions $m_{m}\left(I_{z}, \phi_{z}\right)$ with time dependent amplitudes $\chi_{m}(t)$ as

$\chi_{m}(t) m_{m}\left(I_{z}, \phi_{z}\right)=\int_{0}^{\infty} \mathrm{d} J \int_{0}^{2 \pi} \mathrm{d} \phi(y+i p) \Delta \Psi_{m}\left(\phi_{z}+\omega_{s} t\right)$.

The normalization of $m_{m}$ is done over the initial distribution [20]

$$
\left\langle\overline{m_{m}} m_{m}\right\rangle=\int_{0}^{\infty} \mathrm{d} I_{z} \int_{0}^{2 \pi} \mathrm{d} \phi_{z} \Psi_{0}\left(I_{z}, \phi_{z}\right)\left|m_{m}\left(I_{z}, \phi_{z}\right)\right|^{2}=1,
$$

where the horizontal line implies a complex conjugation.

The evolution of these modes with time is governed by the following equation of motion

$$
\ddot{\chi}_{m} m_{m}+\omega_{m}^{2} \chi_{m} m_{m}=0 .
$$

The eigenmodes $m_{m}$ and eigenfrequencies $\omega_{m}$ can be found with numerical Vlasov solvers, such as DELPHI [14], or circulant matrix solvers, such as BimBim [8].

The wake impulse of mode $m$ can be found by moving the impedance dependent terms to the rhs as

$$
\begin{aligned}
\ddot{\chi}_{m} m_{m}+\omega_{0}^{2} \chi_{m} m_{m} & =\left(\omega_{0}^{2}-\omega_{m}^{2}\right) \chi_{m} m_{m} \\
& =\omega_{0} P_{m}\left(I_{z}, \phi_{z}, t\right) .
\end{aligned}
$$

To drive modes of shape $m_{m}\left(I_{z}, \phi_{z}\right)$, with a discrete frequency shift $\Delta \omega_{m}$, an effective wake kick $P_{m}$ can be found that is proportional to the mode $m_{m}$. It can be evaluated as the average kick on particles starting at $\left(I_{z}, \phi_{z}\right)$, over the synchrotron motion

$$
P_{m}\left(I_{z}, \phi_{z}, t\right)=\left\langle P_{\operatorname{coh}}\left[z\left(t^{\prime}\right), t^{\prime}\right] e^{i \phi_{\Delta m}\left(t^{\prime}\right)}\right\rangle_{t^{\prime}} e^{-i \phi_{\Delta m}(t)},
$$

where the angle brackets signify an average over the subscripted parameter.

\section{Damped eigenmodes $-\boldsymbol{\Omega}_{\boldsymbol{m}}$}

Whether a mode is stabilized by Landau damping or not, is typically assessed with the mapping given by Eq. (1). The equation, which is valid only in the weak head-tail approximation, can be written in terms of frequencies as

$$
\frac{-1}{\Delta \omega_{m j}}=\int_{0}^{\infty} \int_{0}^{\infty} \mathrm{d} J^{2} \frac{J_{j} \frac{\mathrm{d} \Psi\left(J_{x}, J_{y}\right)}{\mathrm{d} J_{j}}}{\Delta \Omega_{m j}-\Delta \omega_{j}\left(J_{x}, J_{y}\right)},
$$

where $\Delta \omega_{m j} \in \mathbb{C}$, is the coherent frequency shift without a frequency spread, $\Delta \omega_{j}=\omega_{j}-\omega_{0 j} \in \mathbb{R}$ is the frequency of an individual particle with actions $\left(J_{x}, J_{y}\right)$, relative to the bare betatron frequency, and $\Delta \Omega_{m j} \in \mathbb{C}$ is the coherent damped frequency shift. The subscript $j$, denoting the plane, will be omitted in the following. The stability diagram is calculated as $\Delta \omega_{m}=\Delta \omega_{\mathrm{SD}}$ in the limit $\operatorname{Im}\left\{\Delta \Omega_{m}\right\} \rightarrow 0^{+}$. If one desires the rise time $\tau_{m}$ of a mode $m$ that is outside/above the stability diagram, this can be found as $\tau_{m}=1 / \operatorname{Im}\left\{\Omega_{m}\right\}>0$ [21].

Obtaining the damping time of a mode $m$ that is inside/ below the stability diagram, as is of interest in this paper, is a greater challenge. The mapping in Eq. (22) has a hole in its domain inside the stability diagram. Van Kampen has addressed this issue in plasma physics, and we will build on his results [22]. Inside the stability diagram, there will exist a continuous spectrum in addition to the discrete modes. However, if the continuous motion is damped faster than the discrete modes, an arbitrary initial distribution may after a short transient time behave like a superposition of damped discrete modes. In the following, we assume that the beam motion can be described sufficiently accurately with a single damped mode. This assumption requires that the continuous spectrum is damped faster than the discrete modes [23]. It will be tested with numerical simulations.

An algorithm is needed to extend the mapping in Eq. (22) inside the stability diagram. The challenge is illustrated in Fig. 1: Find the $\Delta \Omega_{m}$ that corresponds to $\Delta \omega_{m}$. Phrased differently, find an expression relating $M_{\mathrm{coh}}=\Delta \omega_{m}-\Delta \omega_{\mathrm{SD}}$ and $M_{\mathrm{LD}}=\Delta \Omega_{m}-\Delta \Omega_{\mathrm{SD}}$, the margins to the free and damped mode frequencies, for a mode that is barely damped. The free margin $M_{\text {coh }}$ will be chosen to be purely imaginary.

To be physical, the damped frequency shift $\Delta \Omega_{m}$ should change continuously through the artificial discontinuity of the mapping at zero growth rate. If there is no frequency spread, there is no hole in the mapping, and

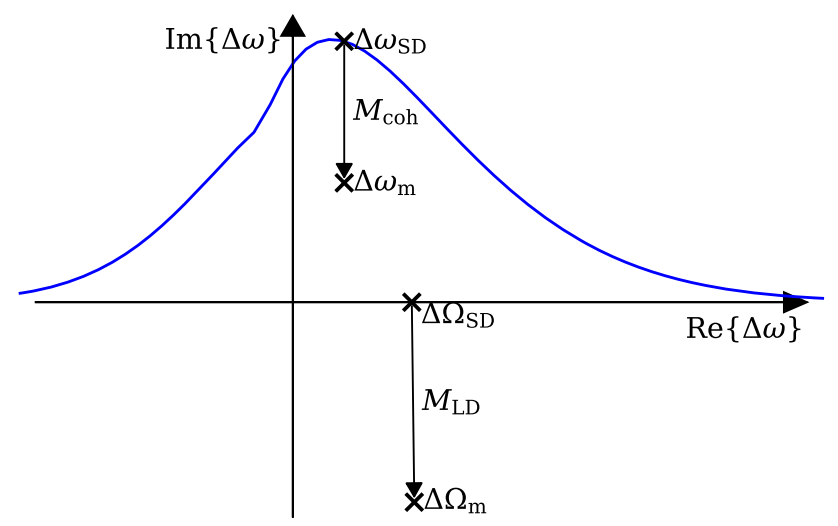

FIG. 1. Illustration of the problem of finding the damped frequency $\Delta \Omega_{m}$ corresponding to the undamped frequency $\Delta \omega_{m}$ that is inside the stability limit in blue, commonly referred to as the stability diagram. 


$$
\frac{-1}{\Delta \omega_{m}}=\frac{-1}{\Delta \Omega_{m}} \Rightarrow M_{\mathrm{LD}}=M_{\mathrm{coh}}
$$

When including a frequency spread, one can do a Taylor expansion, assuming $\left|M_{\text {coh }}\right| \ll\left|\Delta \omega_{\mathrm{SD}}\right|$ and $\left|M_{\mathrm{LD}}\right| \ll\left|\Delta \Omega_{\mathrm{SD}}\right|$. The left-hand side (lhs) becomes

$$
\frac{-1}{\Delta \omega_{m}}=\frac{-1}{\Delta \omega_{\mathrm{SD}}+M_{\mathrm{coh}}}=\frac{-1}{\Delta \omega_{\mathrm{SD}}}\left(1-\frac{M_{\mathrm{coh}}}{\Delta \omega_{\mathrm{SD}}}\right),
$$

where the higher order terms of $M_{\mathrm{coh}} / \Delta \omega_{\mathrm{SD}}$ have been omitted. Doing the same for the integrand on the rhs gives

$$
\frac{1}{\Delta \Omega_{\mathrm{SD}}\left(J_{x}, J_{y}\right)+M_{\mathrm{LD}}}=\frac{1}{\Delta \Omega_{\mathrm{SD}}\left(J_{x}, J_{y}\right)}-\frac{M_{\mathrm{LD}}}{\Delta \Omega_{\mathrm{SD}}\left(J_{x}, J_{y}\right)^{2}},
$$

where $\Delta \Omega_{\mathrm{SD}}\left(J_{x}, J_{y}\right)=\Omega_{\mathrm{SD}}-\omega\left(J_{x}, J_{y}\right)$. After integrating, the first term becomes $-1 / \Delta \omega_{\mathrm{SD}}$, the first term in Eq. (24). Next, assume that the mean squared will be of the same order as the squared mean, $\left\langle 1 / \Delta \Omega_{\mathrm{SD}}^{2}\right\rangle \sim\left\langle 1 / \Delta \Omega_{\mathrm{SD}}\right\rangle^{2}$. Thus, the second terms in Eqs. (24) and (25) gives

$$
\frac{M_{\mathrm{coh}}}{\Delta \omega_{\mathrm{SD}}^{2}}=\frac{1}{\alpha} \frac{M_{\mathrm{LD}}}{\Delta \omega_{\mathrm{SD}}^{2}} \Rightarrow M_{\mathrm{LD}}=\alpha M_{\mathrm{coh}},
$$

where $\alpha$ is a correction factor, which is 1 without a frequency spread as in Eq. (23). Based on this derivation, one cannot determine what $\alpha$ is with detuning, but one can assume that it is close to 1 .

Since the mapping in Eq. (22) works for positive growth rates, one can calculate $\alpha$ in this domain as

$$
\alpha \approx \frac{\partial M_{\mathrm{LD}}}{\partial M_{\mathrm{coh}}}=\lim _{\Delta \Omega_{m} \rightarrow \Delta \Omega_{\mathrm{SD}}} \frac{\Delta \Omega_{m}-\Delta \Omega_{\mathrm{SD}}}{\Delta \omega_{m}-\Delta \omega_{\mathrm{SD}}},
$$

and assume that $\alpha$ varies negligibly for small negative growth rates. In other words, one assumes that $M_{\mathrm{LD}}$ is a smooth function of $M_{\mathrm{coh}}$, in addition to being continuous. The complex $\alpha$, for a Gaussian distribution, is displayed in Fig. 2. For large real frequency shifts, one finds $\alpha \rightarrow 1$, as

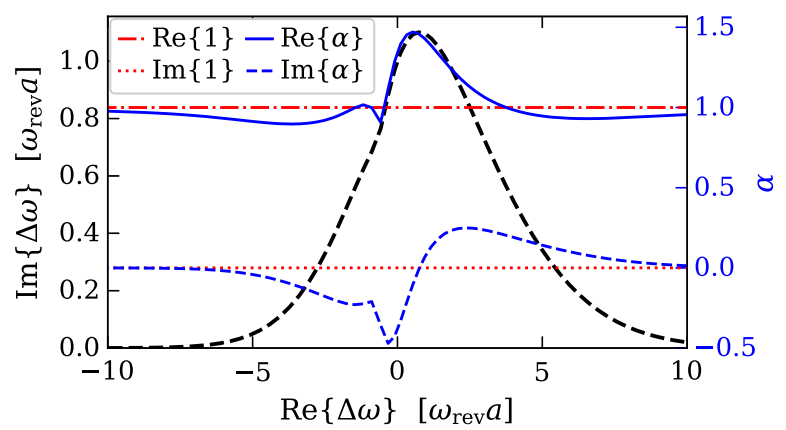

FIG. 2. Stability diagram and $\alpha$ given by Eq. (27), in comparison to the estimate $\alpha=1$. The configuration corresponds to linear detuning coefficients $a=1 \times 10^{-4}$ and $b=-0.7 a$. expected. Equation (26) can be considered a correction to the zeroth order expression in Eq. (23). This expression works well for small $\operatorname{Im}\left\{M_{\text {coh }}\right\}>0$. Our postulate is that it also works for small $\operatorname{Im}\left\{M_{\text {coh }}\right\}<0$, inside the stability diagram. This is tested numerically in Sec. IVA.

\section{Noise excited eigenmodes}

The particle beam in an accelerator is continuously excited by external noise sources. The noise has been neglected in the derivation so far. It can be modeled as an impulse $\tilde{\xi}(t)$ of zero mean, $\langle\tilde{\xi}(t)\rangle_{t}=0$, which can be decomposed as

$$
\tilde{\xi}(t)=\sum_{i} \xi_{i}\left(t_{c}\right) \Xi_{i}(z)
$$

where $\Xi_{i}(z)$ are orthogonal functions of the shape of the noise kick along the bunch, normalized over $\Psi_{0}$, and $\xi_{i}\left(t_{c}\right)$ gives the amplitude of that noise component.

The least stable modes will be excited by the noise. By adding a noise term to the rhs of Eq. (19), using the damped frequency $\Omega_{m}$, multiplying by $\overline{m_{m}}$, and taking the average over the longitudinal distribution, one finds

$$
\ddot{\chi}_{m}+\Omega_{m}^{2} \chi_{m}=\Omega_{m} \sum_{i}\left\langle\overline{m_{m}} \Xi_{i}\right\rangle \xi_{i} .
$$

The excitation per noise component is proportional to the modes' noise moments $\eta_{m i} \in \mathbb{R}$, defined as

$$
\eta_{m i}=\left|\left\langle\overline{m_{m}} \Xi_{i}\right\rangle\right|
$$

The spectrum of the mode amplitudes can be found in the standard way by Fourier transforming Eq. (29)

$$
\mathcal{F}\left(\chi_{m}\right)=\frac{\Omega_{m} \sum_{i}\left\langle\overline{m_{m}} \Xi_{i}\right\rangle \mathcal{F}\left(\xi_{i}\right)}{\operatorname{Re}\left\{\Omega_{m}^{2}\right\}-\omega^{2}+i \operatorname{Im}\left\{\Omega_{m}^{2}\right\}} .
$$

This derivation has modeled the ensemble of oscillators making up the mode as a single damped oscillator with frequency found in Sec. II C. The approximation is valuable, as it allows the analytical latency estimate below in Sec. II F. On the other hand, due to the frequency spread of the individual harmonic oscillators, the frequencies could also be mapped with Eq. (22). That corresponds to a change of $\omega \rightarrow \omega_{\text {SD }}$ and $\Omega_{m} \rightarrow \omega_{m}$ in Eq. (31).

The noise in the LHC is of sufficiently low frequency to change negligibly over the bunch length of 1 ns [24,25]. Therefore, the only noise moment of interest, will be the rigid-bunch (dipolar) moment of the modes $\eta_{m 0}=\left|\left\langle m_{m}\right\rangle\right|$. Dipolar moments can be calculated analytically, e.g. for an airbag bunch using Eq. (6.186) in [17]. However, calculating the dipolar moments for a Gaussian bunch that is perturbed by the transverse feedback system and wakefields will here be done numerically with BimBim. In an 


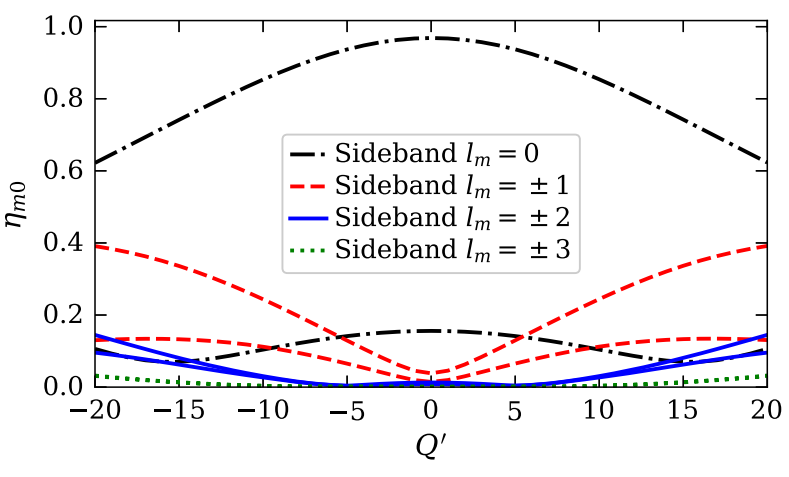

FIG. 3. Largest dipole moments for modes at various sidebands, with various chromaticities and zero transverse feedback. The values have been calculated numerically with BimBim, in the LHC for the experiment described in Sec. V C.

LHC like configuration, but without transverse feedback, the dipole moments of the prevalent modes are presented as a function of the linear chromaticity in Fig. 3. At nonzero chromaticity, the dipolar moment of the angular headtail modes $\left(l_{m} \neq 0\right)$ is nonzero, and can thus be excited by a dipolar noise. These are the most problematic modes in the LHC as of 2018, since the transverse feedback can stabilize the dipolar modes efficiently.

The introduction of crab cavities in an accelerator will introduce crab amplitude noise proportional to $\Xi_{1}=z / \sigma_{z}$ [26]. Headtail modes can have a significant noise moment $\eta_{m 1}$ with this type of noise, already at zero chromaticity.

In the following, we will study the case of white dipolar noise, acceptable over the frequency span of a single bunch. White noise can be characterized by $\left\langle\xi(t) \xi\left(t+t^{\prime}\right)\right\rangle_{t}=\sigma^{2} \delta_{D}\left(t^{\prime}\right)$, where $\delta_{D}(\cdot)$ is the Dirac delta distribution. The impact of the noise on one bunch can be combined into one discrete kick per turn with variance $\sigma_{\text {ext }}^{2}=\tau \sigma^{2}$, where $\tau=1 / f_{\text {rev }}$ is the revolution period of the machine. Thus, the power spectral density of the noise acting on a bunch is effectively given by

$$
S_{\xi}(\omega)=|\mathcal{F}(\xi)|^{2}= \begin{cases}\frac{\sigma_{\mathrm{ext}}^{2}}{\tau}, & \omega \in\left[0, \frac{\omega_{\mathrm{rev}}}{2}\right] . \\ 0, & \text { otherwise }\end{cases}
$$

\section{E. Wakefield driven diffusion}

The noise and wakefields do not only affect the coherent modes, but also the individual particles. Describing the individual particles by their amplitudes $y$ and frequencies $\omega\left(J_{x}, J_{y}\right)$, their motion in one transverse plane is governed by

$$
\begin{aligned}
\ddot{y}+\omega^{2} y & =\omega \xi(t)+\omega P_{\text {wake }} \\
& =\omega \xi(t)+\sum_{m} \frac{\omega}{\omega_{0}}\left(\omega_{0}^{2}-\omega_{m}^{2}\right) \chi_{m} m_{m} .
\end{aligned}
$$

The first term on the lhs models the direct impact of the noise. This term was studied in detail in [9] and found to not be detrimental for stability in the LHC. Thus, it cannot explain the measurements of latent instabilities. The second term is the main focus in this paper, modeling the indirect impact of the noise through the wakefields. Here, the first term will be neglected, and it will be shown that this is an acceptable approximation, since the second term will dominate the diffusion.

To better understand the single-particle dynamics driven by the stochastic force in Eq. (33), it is modeled by a perturbed Hamiltonian as in Eq. (3). Focusing only on the complex effective wake force, the perturbation is given by

$$
\epsilon \mathcal{H}_{1}=-\operatorname{Re}\left\{(y+i p) \overline{P_{\text {wake }}}\right\} \text {, }
$$

which returns the standard perturbation $-y P_{\text {wake }}$ when $P_{\text {wake }} \in \mathbb{R}$ [14-17]. The change of the quickly varying phases due to the perturbation is negligible, while the change of the action is by Hamilton's equations only caused by the perturbation as

$$
\dot{J}=-\frac{\partial \mathcal{H}}{\partial \phi}=-\sqrt{2 J} \operatorname{Re}\left\{i e^{-i \phi} \overline{P_{\text {wake }}}\right\} .
$$

This requires that $\mathcal{H}_{0}$ only depends on the actions, not the phases of the particles.

If the stochastic forces are sufficiently weak to accurately be modeled as a perturbation, they drive a diffusion of the individual particles and thereby the equilibrium distribution $\Psi_{\text {eq }}=\Psi_{0}+\epsilon^{2} \Psi_{2}$. This diffusion can be modeled by $[27,28]$

$$
\begin{gathered}
\frac{\partial \Psi_{\mathrm{eq}}}{\partial t}=\frac{\partial}{\partial J}\left[J D_{\text {wake }} \frac{\partial \Psi_{\mathrm{eq}}}{\partial J}\right] \\
D_{\text {wake }}=\lim _{T \rightarrow \infty} \frac{1}{T} \int_{t_{0}}^{t_{0}+T} \mathrm{~d} t \int_{t_{0}}^{t_{0}+T} \mathrm{~d} s \frac{\langle\dot{J}(t) \dot{J}(s)\rangle_{\phi}}{2 J} .
\end{gathered}
$$

In the interesting regime, the modes are uncoupled. Therefore, the force from the different modes have zero expected correlation, and the diffusion coefficient can be given as

$$
D_{\text {wake }}=\frac{1}{2} \sum_{m} S_{P_{m}}\left[\omega\left(J_{x}, J_{y}\right), I_{z}\right],
$$

where $S_{P_{m}}\left(\omega, I_{z}\right)$ is the power spectral density of the wake force due to mode $m$ on a particle, calculated by combining Eqs. (31)-(33). Since the effective wake force has the same headtail phase shift as the individual particles, these phases will in fact cancel.

In general, the diffusion coefficient in Eq. (38) depends on the longitudinal action $I_{z}$, in addition to the transverse actions, since $S_{P_{m}} \propto\left\langle\overline{m_{m}} m_{m}\right\rangle_{\phi_{z}}$. Because only the transverse 
diffusion is of concern, and the longitudinal distribution is expected to not evolve, the average over $I_{z}$ can be taken, for which the normalization of $m_{m}$ in Eq. (18) is of use. The diffusion coefficient, only dependent on the transverse single-particle frequency $\omega\left(J_{x}, J_{y}\right)$, can be written as

$$
\begin{gathered}
D_{\text {wake }}(\omega)=\sum_{m} D_{\max }^{m} B(\omega) C, \\
D_{\max }^{m}=\frac{\sigma_{\text {ext }}^{2} \eta_{m 0}^{2}\left|\Delta \omega_{m}\right|^{2}}{2 \tau \operatorname{Im}\left\{\Omega_{m}\right\}^{2}}, \\
B(\omega)=\frac{\operatorname{Im}\left\{\Omega_{m}^{2}\right\}^{2}}{\left(\operatorname{Re}\left\{\Omega_{m}^{2}\right\}-\omega^{2}\right)^{2}+\operatorname{Im}\left\{\Omega_{m}^{2}\right\}^{2}}, \\
C=\left|1+\frac{\Delta \omega_{m}}{2 \omega_{0}}\right|^{2} \frac{\left|\Omega_{m}\right|^{2}}{\operatorname{Re}\left\{\Omega_{m}\right\}^{2}},
\end{gathered}
$$

where the noise has been assumed to be dipolar. In the relevant limit of small wake driven frequency shifts $\Delta \omega_{m}, \Delta \Omega_{m} \ll \omega_{0}$, one can ignore terms proportional to the frequency shifts of squared or higher power, and get

$$
\begin{aligned}
B(\omega) & =\frac{\operatorname{Im}\left\{\Omega_{m}\right\}^{2}}{\left(\operatorname{Re}\left\{\Omega_{m}\right\}-\omega\right)^{2}+\operatorname{Im}\left\{\Omega_{m}\right\}^{2}}, \\
C & =1 .
\end{aligned}
$$

The $B$ function, illustrated in Fig. 4, expresses the dependence of the diffusion coefficient on the single-particle frequency $\omega$. It has a maximum value of 1 and half width at half maximum of $\Delta \omega=\left|\operatorname{Im}\left\{\Omega_{m}\right\}\right|$. The $C$ function contains the higher-order terms, and will typically be close to 1 . To express the diffusion coefficient as a function of the tune, one writes $\omega=\omega_{\text {rev }} Q$. Every factor $\omega_{\text {rev }}$ will cancel.

Going from diffusion in 1 transverse plane (1D) to 2 transverse planes (2D) is elementary. The noise is already assumed to be centered, $\langle\xi(t)\rangle_{t}=0$. By further assuming no correlation between the horizontal and vertical noise, and no horizontal wakefields from a vertical offset, there is no cross plane diffusion. Hence, the wakefield driven diffusion equation in $2 \mathrm{D}$ is

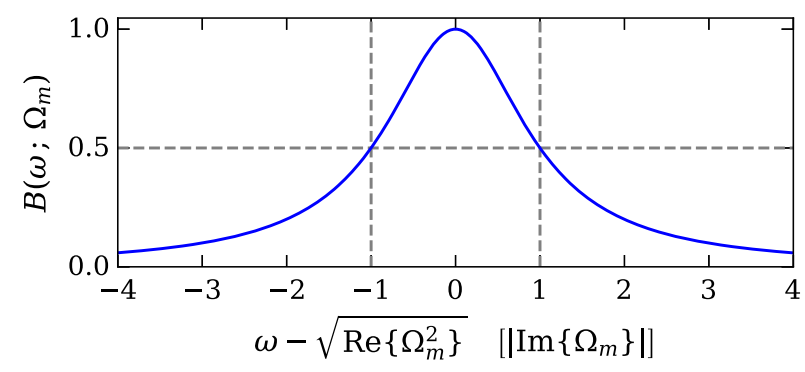

FIG. 4. Shape of diffusion coefficient in frequency space due to a single Landau stabilized mode, given by Eq. (39c). The half width of the half maximum is equal to $\left|\operatorname{Im}\left\{\Omega_{m}\right\}\right|$, the damping rate of the mode.

$$
\begin{aligned}
\frac{\partial \Psi}{\partial t} & =\frac{\partial}{\partial J_{x}}\left[J_{x} D_{x x} \frac{\partial \Psi}{\partial J_{x}}\right]+\frac{\partial}{\partial J_{y}}\left[J_{y} D_{y y} \frac{\partial \Psi}{\partial J_{y}}\right], \\
D_{j j}\left(J_{x}, J_{y}\right) & =D_{\text {wake } j}\left[\omega_{\text {rev }} Q_{j}\left(J_{x}, J_{y}\right)\right],
\end{aligned}
$$

where $D_{\text {wakej }}$, given in Eq. (39), includes the noise and details of the modes in the transverse plane $j$. Since Eq. (40) is linear, one can to first order simply add other types of diffusion, such as the direct diffusion driven by the external noise, diffusion due to incoherent noise, or diffusion due to chaotic regions in phase space, most prevalent at high actions.

\section{F. Instability latency}

The diffusion derived in Sec. II E will locally flatten the distribution around the actions resonant with the frequency of the least stable mode. Since the derivative of the distribution appears in the dispersion integral in Eq. (22), a change of the stability diagram is expected. In general, the diffusion modeled by Eq. (37) requires a numerical technique to be solved accurately, as will be done in Sec. III. Nevertheless, the latency can be calculated analytically under certain assumptions, as will be done here. For ease of notation, the complex frequencies will, in this subsection only, be written with an alternative notation as

$$
\begin{aligned}
& \omega=\operatorname{Re}\{\omega\}+i \operatorname{Im}\{\omega\}=\tilde{\omega}+i \gamma, \\
& \Omega=\operatorname{Re}\{\Omega\}+i \operatorname{Im}\{\Omega\}=\tilde{\Omega}+i \Gamma,
\end{aligned}
$$

using the subscripts already introduced in Fig. 1. Furthermore, only horizontal noise, and thus diffusion, will be considered, and it is assumed that only one dominant mode drives the diffusion.

In $1 \mathrm{D}$, the stability diagram can be calculated as [29]

$$
\begin{aligned}
\lim _{\epsilon \rightarrow 0^{+}} \int_{-\infty}^{\infty} \frac{f(x) \mathrm{d} x}{g(x)+i \epsilon} & =\mathrm{P} \int_{-\infty}^{\infty} \frac{f(x) \mathrm{d} x}{g(x)}-i \pi \int_{-\infty}^{\infty} f(x) \delta_{D}[g(x)] \mathrm{d} x \\
& =\mathrm{P} \int_{-\infty}^{\infty} \frac{f(x) \mathrm{d} x}{g(x)}-i \pi \sum_{\substack{x_{r} \text { s.t. } \\
g\left(x_{r}\right)=0}} \frac{f\left(x_{r}\right)}{\left|g^{\prime}\left(x_{r}\right)\right|},
\end{aligned}
$$

where $f(x), g(x) \in \mathbb{R}, \mathrm{P}$ denotes the principal value, $g^{\prime}(x)=\partial_{x} g(x)$, and the sum is over all zeros $x_{r}$ of the function $g(x)$.

The penultimate goal of this section is to calculate the imaginary part of the stability diagram, $\gamma_{\mathrm{SD}}\left(\tilde{\omega}_{m}\right)=\gamma_{\mathrm{SD} r}$, at the real frequency $\tilde{\omega}_{m}$ of the least stable mode. With a $2 \mathrm{D}$ frequency spread, it is

$$
\frac{\gamma_{\mathrm{SD} r}}{\left|\Delta \omega_{\mathrm{SD} r}\right|^{2}}=-\pi \int_{0}^{\infty} \int_{0}^{\infty} \mathrm{d} J^{2} J_{x} \Psi^{\prime} \delta_{D}\left[\tilde{\Omega}_{\mathrm{SD} r}-\omega\left(J_{x}, J_{y}\right)\right],
$$

where $\Psi^{\prime}=\partial \Psi /\left.\partial J_{x}\right|_{\left(J_{x}, J_{y}\right)}$. Consider linear detuning, as given by Eq. (9). Assuming that $\tilde{a}$ is nonzero, there will for 
each vertical action be only one resonating horizontal action $J_{x r}$, defined by

$$
\Delta \tilde{\Omega}_{\mathrm{SD} r}=\tilde{a} J_{x r}\left(J_{y}\right)+\tilde{b} J_{y} .
$$

Furthermore, in the limit of large relative real tune shifts $\left|\Delta \tilde{\omega}_{\mathrm{SD} r}\right| \gg\left|\gamma_{\mathrm{SD} r}\right|$, which is true for the least stable modes in the LHC, and assuming that the real part changes negligibly, since $\Psi^{\prime}$ only will change close to the resonance, the imaginary part can be approximated by

$$
\frac{\gamma_{\mathrm{SD} r}}{\left|\Delta \tilde{\omega}_{\mathrm{SD} r}\right|^{2}}=-\frac{\pi}{|\tilde{a}|} \int_{0}^{\infty} \int_{0}^{\infty} \mathrm{d} J^{2} J_{x} \Psi^{\prime} \delta_{D}\left[J_{x}-J_{x r}\left(J_{y}\right)\right] .
$$

The imaginary part of the stability diagram, at the critical frequency, is under these assumptions proportional to the derivative of the distribution at the resonant actions. Thus, the local flattening of the distribution will lead to a reduction of $\gamma_{\mathrm{SD} r}$, which can be illustrated as the drilling of a borehole in the stability diagram at this critical frequency. See a visual illustration of this process in Fig. 8. If this process carries on for long enough uninterrupted, it can lead to a loss of Landau damping as $\gamma_{\mathrm{SD} r} \rightarrow \gamma_{m}^{+}$. Note that in the limit of small relative real tune shifts, $\left|\Delta \tilde{\omega}_{\mathrm{SD} r}\right| \ll\left|\gamma_{\mathrm{SD} r}\right|, \gamma_{\mathrm{SD} r}$ would be proportional to the inverse of the rhs of Eq. (44), and a local flattening would initially lead to an increase of the imaginary part of the stability diagram. This special case will be investigated in more detail numerically in Sec. VA.

To evaluate the evolution of $\gamma_{\mathrm{SD} r}$, one must evaluate the evolution of $\Psi^{\prime}$. Taking the action derivative of the diffusion equation in Eq. (40) gives

$$
\frac{\partial \Psi^{\prime}}{\partial t}=\frac{\partial^{2}}{\partial J_{x}}\left[J_{x} D_{x x} \Psi^{\prime}\right]
$$

It is only the evolution of $\Psi^{\prime}$ at the center of the diffusion coefficient in Fig. 4 that is of interest. However, this evolution also depends on the diffusion close to it. To evaluate the macrodiffusion, the finite difference of the rhs of Eq. (45) has been taken at $\left\{J_{x r}-W_{J}, J_{x r}, J_{x r}+W_{J}\right\}$, where $W_{J}=\left|\Gamma_{m} / \tilde{a}\right|$ is the half width at half maximum in the horizontal action coordinate. By assuming a Gaussian initial distribution in $(y, p)$ phase space, equal to an exponential distribution in action $\Psi_{0}\left(J_{x}, J_{y}\right)=e^{-J_{x}-J_{y}}$, Eq. (45) becomes

$$
\frac{1}{\Psi_{r}^{\prime}} \frac{\partial \Psi_{r}^{\prime}}{\partial t}=-\frac{D_{\max }}{W_{J}^{2}}\left[J_{x r}+W_{J}^{2}-J_{x r} W_{J}^{2}+\mathcal{O}\left(W_{J}^{3}\right)\right],
$$

where $\Psi_{r}^{\prime}=\Psi^{\prime}\left(J_{x r}\left(J_{y}\right), J_{y}\right)$. For the relevant cases, it has already been stated that $\left|\Delta \tilde{\omega}_{\mathrm{SD} r}\right| \gg\left|\gamma_{\mathrm{SD} r}\right|$, and that the mode is close to the stability threshold, $\gamma_{m} \lesssim \gamma_{\mathrm{SD} r}$. Furthermore, it is illustrated in Fig. 2 that $\max \left(\gamma_{\mathrm{SD} r}\right) \approx \tilde{a}$, when $\tilde{b}$ is small wrt to $\tilde{a}$. It follows that $W_{J} \ll 1$. Hence, the first term on the rhs of Eq. (46) will dominate, except when $J_{x r} \approx 0$. If $\tilde{b} \neq 0$, then $J_{x r}\left(J_{y}\right)$ is not constant, and one can calculate an effective action, as will be done in the following. Therefore, all but the first term on the rhs will be neglected.

If the rhs of Eq. (46) would have been constant, the distribution derivative would have evolved as

$$
\Psi_{r}^{\prime}(t)=\Psi_{r}^{\prime}(0) \exp \left(-\frac{J_{x r} D_{\max }}{W_{J}^{2}} t\right)
$$

where the flattening would gradually slow down. This is not correct. As $\Psi_{r}^{\prime}$ is flattened, $\gamma_{\mathrm{SD} r}$ is reduced while $\gamma_{m}$ stays put, such that $\Gamma_{m}$ approaches 0 and $D_{\max } / W_{J}^{2}$ increases. This evolution has to be solved self-consistently.

The ultimate goal of this section is to get the latency, i.e., the time it takes for $\gamma_{\mathrm{SDr}} \rightarrow \gamma_{m}^{+}$and $\Gamma_{m} \rightarrow 0^{-}$. Combining Eqs. (44) and (46), returns an expression for the relative time derivative of $\gamma_{\mathrm{SD} r}$

$$
\begin{gathered}
\frac{1}{\gamma_{\mathrm{SD} r}} \frac{\partial \gamma_{\mathrm{SD} r}}{\partial t}=-\frac{J_{x, \mathrm{eff}} D_{\max }}{W_{J}^{2}}, \\
J_{x, \text { eff }}=\frac{\int_{0}^{\infty} \int_{0}^{\infty} \mathrm{d} J^{2} J_{x}^{2} \Psi^{\prime} \delta_{D}\left[J_{x}-J_{x r}\left(J_{y}\right)\right]}{\int_{0}^{\infty} \int_{0}^{\infty} \mathrm{d} J^{2} J_{x} \Psi^{\prime} \delta_{D}\left[J_{x}-J_{x r}\left(J_{y}\right)\right]},
\end{gathered}
$$

using that only $\Psi^{\prime}$ depends on time. Assuming that $\tilde{a} \tilde{b} \leq 0$ and $\tilde{a} \neq 0$, as in the LHC, one gets that

$J_{x, \text { fff }}=\min \left(J_{x r}\right)+\frac{b}{b-a}+\frac{\left(\frac{b}{b-a}\right)^{2}}{\min \left(J_{x r}\right)+\frac{b}{b-a}} \geq \frac{2 b}{b-a}$,

expressed in $a$ and $b$, as the factors $\omega_{\text {rev }}$ have canceled out. The value $\min \left(J_{x r}\right)$ is the minimum horizontal action of a resonant particle, being equal to $\max \left\{0, \Delta \tilde{\Omega}_{\mathrm{SD} r} / \tilde{a}\right\}$. The most critical modes in the LHC have $\Delta \tilde{\Omega}_{\mathrm{SD} r}<0$. For a positive octupole current, $\tilde{a}>0$, one then gets $\min \left(J_{x r}\right)=0$. For a negative octupole current, on the other hand, one gets $\min \left(J_{x r}\right)>0$ and the effective action $J_{x \text {,eff }}$ will be larger.

To get the evolution of $\Gamma_{m}$, one combines Eqs. (47) and (26), assuming that $\alpha$ will stay constant. This assumption is a simplification of the physics, necessary to reach an analytical expression for the latency, and it is the main difference in this derivation from what the numerical solver can model. Since $\gamma_{m}$ is constant, it follows that $\partial_{t} \Gamma_{m}=-\operatorname{Re}\left\{\alpha_{0}\right\} \partial_{t} \gamma_{\mathrm{SD} r}$. Thus, one gets

$\frac{1}{\operatorname{Re}\left\{\alpha_{0}\right\} \gamma_{\mathrm{SD} r}} \frac{\partial \Gamma_{m}}{\partial t}=\frac{J_{x, \text { eff }} D_{\max }}{W_{J}^{2}}=\frac{J_{x, \text { eff }} D_{\max 0} \Gamma_{m 0}^{2} \tilde{a}^{2}}{\Gamma_{m}^{4}}$,

where the subscript 0 denotes that the values should be taken at time $t=0$, when the diffusion process starts. Note that $\Gamma_{m}<0$ when stable, such that a positive gradient takes 
it toward 0 and a possible loss of Landau damping. The latency $L$ can finally be calculated as

$$
L=\frac{1}{J_{x, \mathrm{eff}} D_{\max 0} \Gamma_{m 0}^{2} \tilde{a}^{2}} \int_{\Gamma_{m 0}}^{0} \frac{\Gamma_{m}^{4} \mathrm{~d} \Gamma_{m}}{\operatorname{Re}\left\{\alpha_{0}\right\} \gamma_{m}-\Gamma_{m}},
$$

where the fraction outside the integral is a constant only dependent on the initial condition.

The latency integral in Eq. (50) can be approximated as the integrand is always positive and its denominator goes from $\operatorname{Re}\left\{\alpha_{0}\right\} \gamma_{\mathrm{SD} r 0}$ to $\operatorname{Re}\left\{\alpha_{0}\right\} \gamma_{m}$, which should be a small relative change according to the assumptions. A lower estimate can be calculated by assuming the initial value, and a maximum value can be calculated by assuming $\gamma_{m}=0$

$$
\frac{\left(\gamma_{\mathrm{SD} r 0}-\gamma_{m}\right) W_{J 0}^{2}}{5 \gamma_{\mathrm{SD} r 0} J_{x, \mathrm{eff}} D_{\max 0}} \equiv L_{L} \leq L \leq L_{L} \frac{5}{4} .
$$

Inserting the physical quantities gives

$$
\begin{aligned}
\frac{L_{L}}{\tau} & =\frac{\gamma_{\mathrm{SD} r 0}-\gamma_{m}}{\gamma_{\mathrm{SD} r 0}} \frac{\left(\gamma_{\mathrm{SD} r 0}-\gamma_{m}\right)^{4}}{2.5 \tilde{a}^{2}\left|\Delta \omega_{m}\right|^{2}} \frac{\operatorname{Re}\left\{\alpha_{0}\right\}^{4}}{J_{x, \mathrm{eff}} \sigma_{\mathrm{ext}}^{2} \eta_{m 0}^{2}} \\
& =\frac{\left(\operatorname{Im}\left\{\Delta Q_{\mathrm{SD} r 0}-\Delta Q_{\mathrm{coh}}\right\}\right)^{5}}{2.5 \operatorname{Im}\left\{\Delta Q_{\mathrm{SD} r 0}\right\} a^{2}\left|\Delta Q_{\mathrm{coh}}\right|^{2}} \frac{\operatorname{Re}\left\{\alpha_{0}\right\}^{4}}{J_{x, \mathrm{eff}} \sigma_{\mathrm{ext}}^{2} \eta_{m 0}^{2}} .
\end{aligned}
$$

As $\tau$ is the revolution period in the machine, the rhs of Eq. (52) gives the lower estimate of the latency in number of turns. Remember that $\Delta Q_{\mathrm{SD} r 0}$ is the point on the stability diagram at the same real tune as the coherent mode, $\operatorname{Re}\left\{\Delta Q_{\mathrm{SDr} 0}\right\}=\operatorname{Re}\left\{\Delta Q_{\text {coh }}\right\}$. Furthermore, note that $\alpha_{0}$ to zeroth order can be approximated by $\alpha_{0}=1$, as illustrated in Fig. 2. This expression can serve as a guide in designing future hadron accelerators to avoid this diffusion driven instability mechanism.

The latency, according to this analytical calculation, is proportional to $W_{J 0}^{2} / J_{x, \text { eff }} D_{\max 0}$. In words, the latency is shorter for a fast diffusion that is narrow in action space. Such a diffusion will efficiently reduce $\Psi^{\prime}$ at the resonant actions. The latency is also proportional to $\left(\gamma_{\mathrm{SD} r 0}-\gamma_{m}\right) / \gamma_{\mathrm{SD} r 0}$. This is a measure on how much the stability diagram must be lowered, for the mode to reach the stability threshold. At first glance, it may look like the latency is shorter for a larger detuning coefficient $(a)$, which would be counterintuitive, but note that a larger $a$ also leads to a more negative $\Gamma_{m}$, which in total leads to a longer latency.

\section{NUMERICAL METHOD}

The diffusion modeled by Eq. (36) must be solved numerically to be solved accurately. Numerical estimates are also needed to lift the assumptions, such as the one of large real tune shifts, taken in Sec. II F. The results that will be presented in the following have been produced with a finite volume method (FVM) solver implemented in PyRADISE [9] (PYTHON radial diffusion and stability evolution). The 2D transverse action space has been discretized into a $700 \times 700$ grid, going from 0 to $J_{\max }=20$, which is equidistant in $\sqrt{J}$. Thus, the minimal grid spacing is $\min (\Delta J)=4 \times 10^{-5}$, and the grid spacing increases for larger actions where the distribution is less dense.

It has been assumed that a single mode is dominant, although this is not a requirement of the code. The diffusion coefficient has been recalculated at each time step during the diffusion process, whereupon the diffusion has been solved with an implicit backward differentiation formula for the following time step. Using an implicit scheme is necessary, as the maximum value of the diffusion coefficient will grow as the instability is approached, eventually rendering an explicit solver numerically unstable.

The FVM solver gives as output the evolving distribution $\Psi_{k}$ after each discrete time step $t_{k}$. For each of these distributions, the tune $Q_{\mathrm{LD}}$ of the damped mode is calculated using the algorithm in Sec. II C and a numerical trapezoidal integrator implemented in PySSD [30], which has been imported in PyRADISE. If $\operatorname{Im}\left\{Q_{\mathrm{LD}}\right\}$ eventually becomes positive, the bunch will be considered to have become unstable.

\section{NUMERICAL VERIFICATION}

In this section, various aspects of the new theory derived in Sec. II will be tested with macroparticle simulations run in COMBI $[8,31]$.

\section{A. Dynamics inside the stability diagram}

The response of the beam to an external noise has been modeled as a single under-damped harmonic oscillator with a complex frequency, found by Eqs. (26) and (27). To verify this, simulations have been run with $10^{6}$ macroparticles. The simulations were run with an antidamper inducing a complex tune shift $\Delta Q_{\text {coh }}=-1.47 \times 10^{-4}+1.25 \times 10^{-5} i$. This mode is stabilized with $a=a_{\text {thresh }}=5.0 \times 10^{-5}$ and $b=-0.7 a$. The real and imaginary tune shifts, in both the simulations and according to Eqs. (26) and (27), are presented in Fig. 5. Each point is the average of 10 simulations, from which the standard deviation has been used as an error bar. The Taylor algorithm works equally well for modes outside and inside the stability diagram. For this complex tune shift, the approximation $\alpha=1$ has a minimal impact close to $a_{\text {thresh }}$, compared to finding $\alpha$ by Eq. (27).

The simulations presented here were initialized with nonzero initial horizontal amplitudes, to get a measurable evolution from which to calculate the negative imaginary frequency. With a nonzero initial offset, some bunches that were supposed to be barely stabilized by Landau damping, eventually became unstable. This was caused by a similar mechanism to the one introduced in this paper, except that the noise over multiple turns was combined into a single kick. Furthermore, according to the law of large numbers, the average of $N_{p}$ numbers drawn from a centered Gaussian distribution of spread $\sigma_{x}$, will itself be drawn from a Gaussian distribution with zero mean and spread 

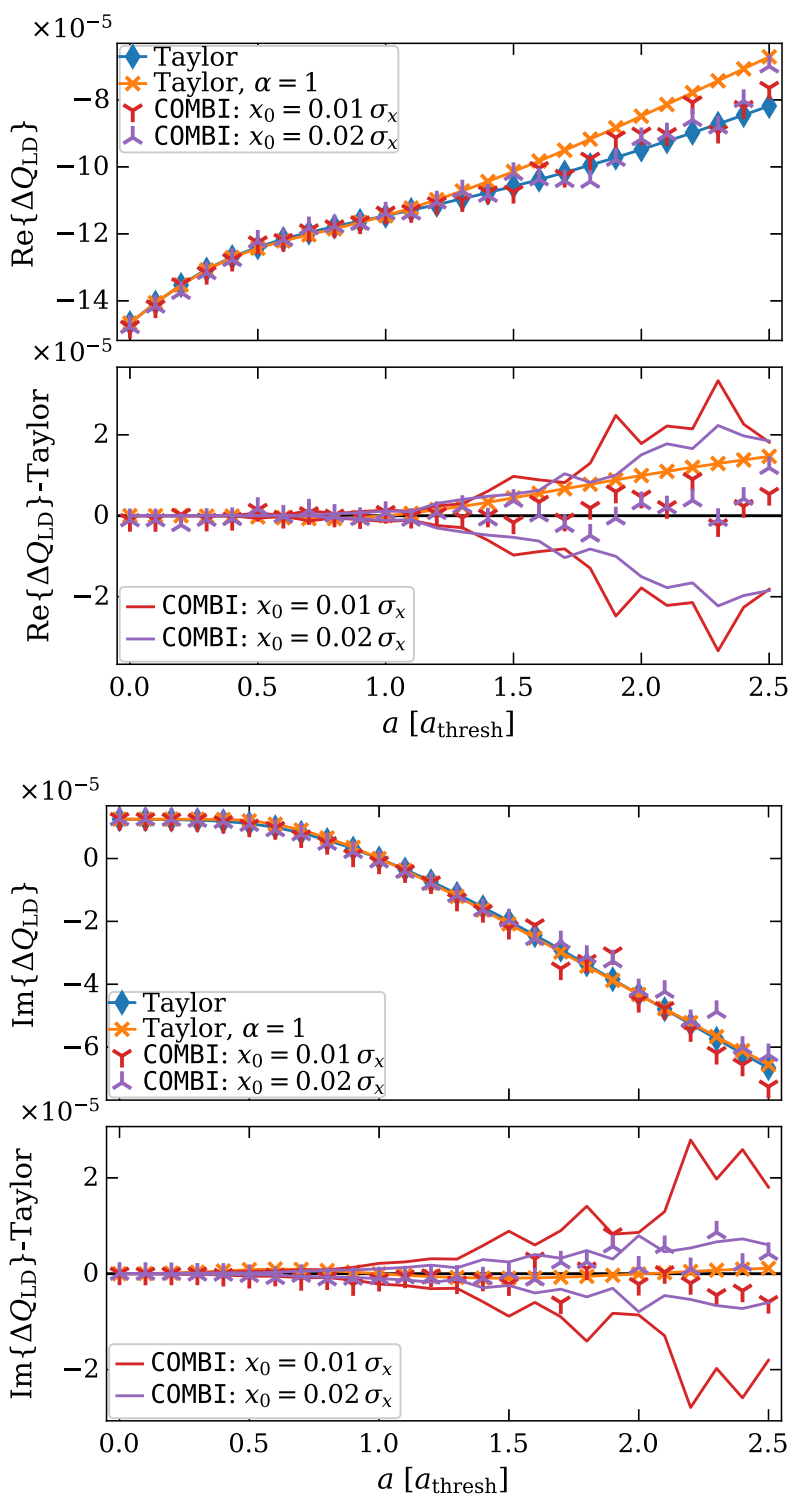

FIG. 5. Tune shift of a coherent mode outside and inside the stability diagram. The theoretical curves have been calculated with Eq. (26), using both $\alpha=1$ and $\alpha$ given by Eq. (27). The error margin (solid lines in the bottom) is the standard deviation (std) of 10 simulations, for each value of $a$ separately.

$\sigma_{x} / \sqrt{N_{p}}$. Therefore, the imaginary part of the frequency has been calculated based on the initial damping until the center of mass reached $4 \times 10^{-3} \sigma_{x}$, which occurs earlier for a larger $a$ and smaller initial offset, causing a larger error bar at these points. The real part of the frequency has been calculated based on the turn-by-turn position, using an interpolated fast Fourier transform implemented in the code HARPY [32]. The real part did not change systematically with time for each calculation separately.

\section{B. Wakefield driven diffusion}

In Sec. II, the impact of the wakefields was modeled as a diffusion, with diffusion coefficient given by Eq. (39).
Here, we want to verify this expression, by comparing to a numerical diffusion coefficient

$$
D_{\text {num }}\left(J_{0}\right)=\frac{\left\langle\Delta J^{2}\right\rangle}{2 T J_{0}}
$$

where $J_{0}$ is the initial action of a particle and $\Delta J$ is the change of action after $T$ turns. Without loss of generality, the diffusion coefficient is in this section given in units of action variance per turn, instead of per second.

The test configuration includes an antidamper induced tune shift $\Delta Q_{\mathrm{coh}}=-1.47 \times 10^{-3}+1.25 \times 10^{-4} i$, $a_{\text {thresh }}=5 \times 10^{-4}, \quad a=1.5 a_{\text {thresh }}, \quad b=-0.7 a, \quad Q^{\prime}=0$, and $\sigma_{\mathrm{ext}}=1 \times 10^{-4}$. 20 simulations have been run with $N_{p}=10^{7}$ particles over $T=10^{4}$ turns. This many particles were needed to make the numerical noise negligible, and this few turns were necessary to avoid a significant change of the distribution and thereby the stability. The individual and average numerical diffusion coefficients are presented in Fig. 6(a) in comparison to Eq. (39). The numerical diffusion coefficients in the individual simulations have not reached the expectancy value, but the average has. The apparent small difference in the real tune shift of the coherent mode may be

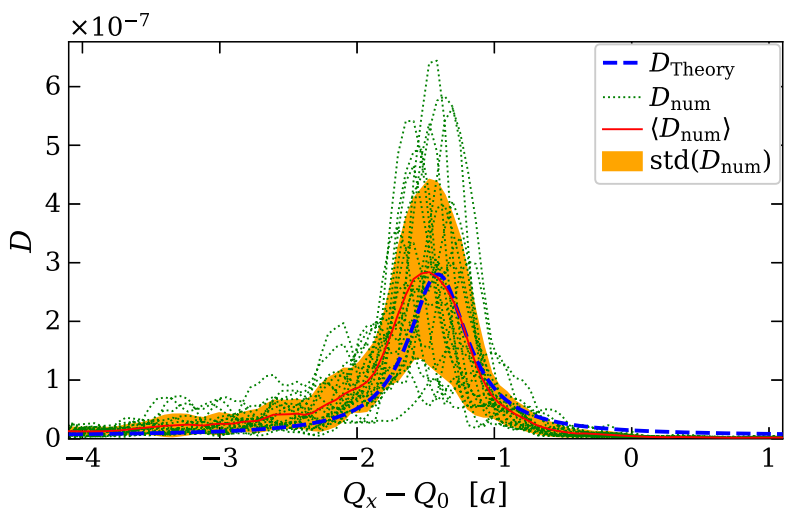

(a)

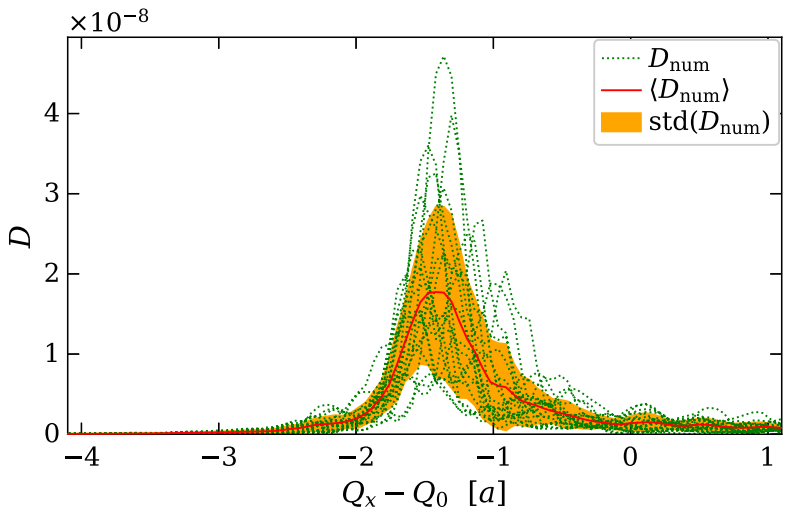

(b)

FIG. 6. Numerical diffusion coefficient in 20 simulations and comparison to theory given by Eq. (39). (a) with external noise of amplitude $\sigma_{\text {ext }}=10^{-4}$. (b) without external noise. 
attributed to the Taylor technique used to map $\Delta Q_{\text {coh }}$ to $\Delta Q_{\mathrm{LD}}=-1.06 \times 10^{-3}-1.987 \times 10^{-4} i$. The width of the average numerical diffusion coefficient is slightly larger than in the new theory, which may cause an underestimation of the latency, analytically found to be proportional to the width squared in Eq. (51). Note that the peak wake diffusion $\sim 3 \times 10^{-7}$ /turn is significantly larger than the direct diffusion $5 \times 10^{-9} /$ turn, as stated in Sec. IIE. The direct diffusion will be reduced even further in the presence of a transverse feedback [9].

In the simulations presented in this section, we have used the antidamper, equal to an ideal dipolar impedance, acting on the centroid of the beam. This way, the coherent tune shift $\Delta Q_{\text {coh }}$ can be controlled perfectly. The main challenge in calculating $D_{\text {num }}$ is that a bunch consisting of $N_{p}$ particles normally distributed with zero mean and spread $\sigma_{x}$, will not actually have zero mean, due to the law of large numbers. Therefore, a large number of particles is needed for the numerical noise to be negligible compared to the small controlled external noise. With $10^{7}$ particles, the numerical diffusion coefficient without external noise is presented in Fig. 6(b). The maximum of this average is $6 \%$ of the maximum with external noise. At least this many particles is therefore needed in simulations of this configuration.

\section{Diffusion or resonant motion?}

It was assumed in Sec. IIE that the impact of the noise excited wakefields was sufficiently stochastic to be modeled as a diffusion mechanism. Whether the events in the future and the past are sufficiently independent was considered by a phase mixing condition in [28]. The autocorrelation function of the underdamped stochastic harmonic oscillator (USHO) is an exponential $\exp \left(\operatorname{Im}\left\{\Omega_{m}\right\}\left|t-t^{\prime}\right|\right)$, which satisfies the phase mixing condition. The process will in this case converge to a diffusion process in the limit of small perturbations $\epsilon \mathcal{H}_{1} \rightarrow 0$ and long times $t \rightarrow \infty$. Thus, a minimal requirement is a lower limit on the time of the process, which should be much longer than the correlation time of the USHO, $L \gg \tau_{m}=1 /\left|\operatorname{Im}\left\{\Omega_{m}\right\}\right|$. For typical modes in the LHC, this requirement implies that the latency should be $L \gg 10$ s to be well modeled as a diffusion.

Now, consider in more detail the process at hand. To be modeled as a diffusion, the change of the distribution during a correlation time of the stochastic excitation must be limited. Therefore, the integrated change of a particle's action due to the wake force within one correlation time, $\kappa_{\text {wake }}$, must be small compared to the width of the diffusion coefficient in action space. The power spectral density of the noise excited wakefields was in Eq. (39) found to have half width at half maximum of $\left|\operatorname{Im}\left\{\Omega_{m}\right\}\right|$. Since the considered detuning is caused by octupoles, the width in action space is given by $W_{J}=\left|\operatorname{Im}\left\{\Omega_{m}\right\}\right| / \tilde{a}$. In the relevant limit of a small $\Delta \omega_{m}$ and a single dominating mode, one can write $P_{\text {wake }}=P_{m}=-2 \Delta \omega_{m} \chi_{m} m_{m}$. The mean square amplitude of an USHO can be given as the product of the noise power spectral density and $\tau_{m} / 4$. Thus, the condition for diffusion can be written as

$$
\kappa_{\text {wake }} \approx \frac{\eta_{m 0} \sigma_{\mathrm{ext}}\left|\Delta \omega_{m}\right|}{\left|\operatorname{Im}\left\{\Omega_{m}\right\}\right|^{3 / 2}} \ll\left|\frac{\operatorname{Im}\left\{\Omega_{m}\right\}}{\tilde{a}}\right| .
$$

Due to the definition of $\sigma_{\mathrm{ext}} \propto \sqrt{\tau}$, there is no direct dependence on the revolution frequency.

In the limit $\operatorname{Im}\left\{\Omega_{m}\right\} \rightarrow 0$, the wake force becomes a deterministic harmonic excitation of a single frequency. The particles will oscillate around the harmonic frequency $\operatorname{Re}\left\{\Omega_{m}\right\}$, causing an oscillation of the distribution and emittance, discussed in more detail in Appendix. The maximum half width in action of particles oscillating around the harmonic frequency is $W_{h}$, estimated by Eq. (A14). The period of this oscillation is estimated to be somewhat larger than the minimal $\tau_{h \min }$, given by Eq. (A12). This deterministic evolution will be negligible when $W_{h} \ll W_{D}$ and $\tau_{h \min } \gg \tau_{m}$. Both these requirements and the requirement in Eq. (54) can be summarized by a condition on a single ratio of relevant parameters

$$
R_{D}=\frac{\left|\operatorname{Im}\left\{\Delta \Omega_{m}\right\}\right|^{2.5}}{a f_{\text {rev }} \sigma_{\text {ext }} \eta_{m 0}\left|\Delta \omega_{m}\right|} \gg 1
$$

If this condition is met, then the diffusion will dominate over the resonant motion.

The condition on $R_{D}$ has been estimated by simple calculations. Simulations have been run to verify this condition, and to find how large $R_{D}$ needs to be, before the process is acceptably estimated by a diffusion. The emittance evolution in 5 different simulations is presented in Fig. 7(a) and the distribution evolution for the case of $R_{D}=10$ is presented in Fig. 7(b). It was assumed in Sec. II that the least stable mode could be modeled as an USHO. To avoid the numerical noise challenges described in Sec. IV B, such a stochastic oscillator has been implemented in COMBI. There is a great agreement between the simulations and the diffusion solver PyRADISE for $R_{D}=10$. For small values of $R_{D}$, the emittance oscillates as in the Appendix and is clearly dominated by the resonance. It seems that the evolution is fairly well modeled as a diffusion starting already at $R_{D}=1$. In all the following results, it has been checked that $R_{D} \gg 1$ at the initial condition.

The result in this section is that the effect of the noise excited wakefields is a diffusion only if the noise is small enough. In Sec. IV B, it was found that a large noise was needed to be stronger than the numerical noise. To model this mechanism self-consistently with macroparticle simulations, both of these requirements must be met. One should use a minimum of $10^{7}$ macroparticles and $\mathcal{O}\left(10^{6}\right)$ turns. 


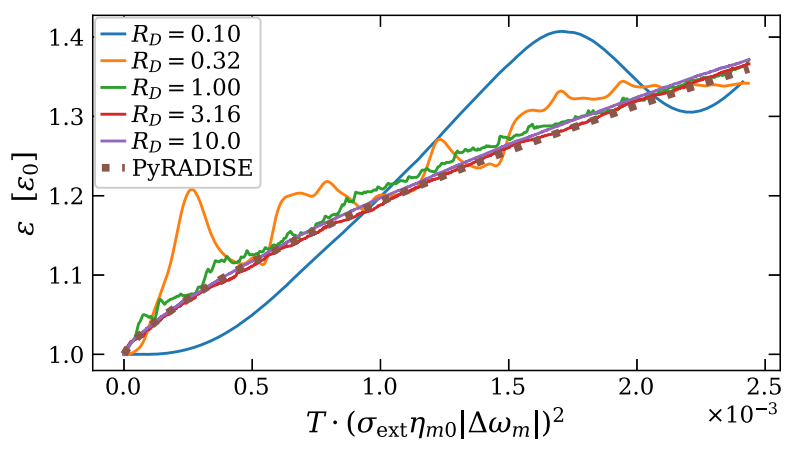

(a)

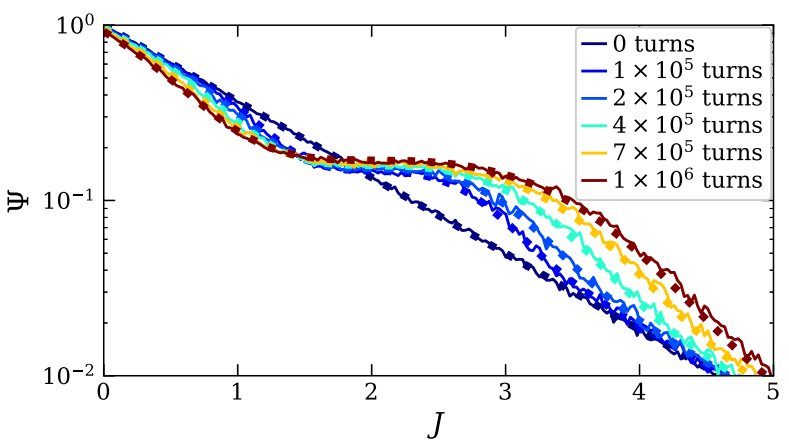

(b)

FIG. 7. Evolution of emittance in (a) for different values of $R_{D}$ given by Eq. (55), and of the distribution in (b) for $R_{D}=10$. The solid lines correspond to macroparticle simulations in COMBI, while the dotted lines have been calculated with PyRADISE. $R_{D}$ was changed by changing the external noise amplitude $\sigma_{\text {ext }}$ and the number of turns $T$, while keeping the product $T \sigma_{\mathrm{ext}}^{2}$ constant.

This is why the analytical diffusion model presented in this paper is invaluable in evaluating this mechanism.

\section{RESULTS}

\section{A. Distribution and stability evolution}

In this section we will display the change of the distribution, and corresponding change of the stability diagram, in two representative configurations with only horizontal noise. Both cases use $a_{\text {thresh }}=5 \times 10^{-5}, a=1.5 a_{\text {thresh }}, b=-0.7 a$, and $\eta_{m 0} \sigma_{\text {ext }}=1 \times 10^{-4}$. The difference is the coherent tune shift in absence of Landau damping, $\Delta Q_{\text {coh. Note that the }}$ diffusion coefficient in Eq. (39) and analytical latency estimate in Eq. (50) does not depend on the absolute values of $a$ and $\Delta Q_{\text {coh }}$, only on the ratio between them. This fact is also illustrated in Fig. 2, as the frequency shifts are given in units proportional to $a$.

In the first case, the least stable mode is at $\Delta Q_{\text {coh }}=-1.47 \times 10^{-4}+1.25 \times 10^{-5} i$. This is the same configuration as was tested in Sec. IVA. The relative change of the distribution at the time of the instability is illustrated in Fig. 8(a). The distribution is locally flattened in the horizontal direction at the resonant actions $Q\left(J_{x}, J_{y}\right)=Q_{\mathrm{LD} x}$, equivalently to the process illustrated in Fig. 7 . The local

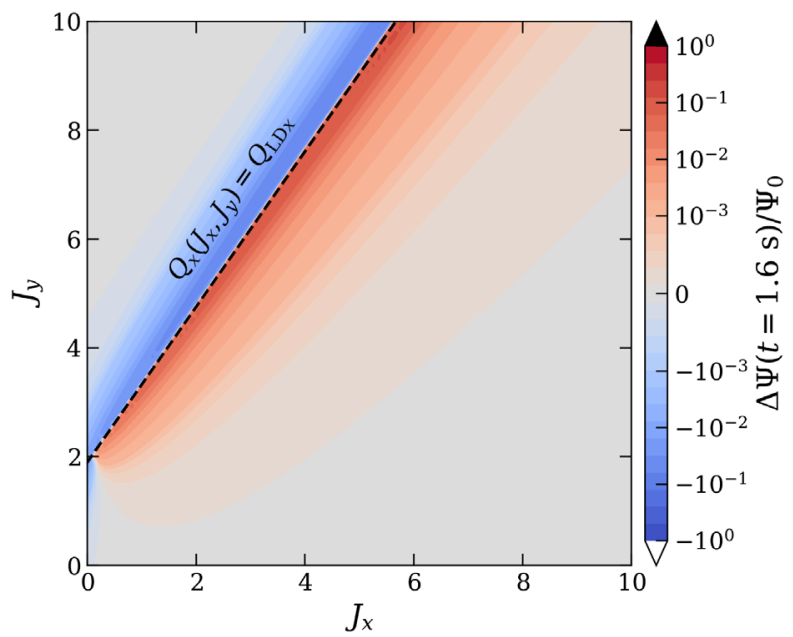

(a)

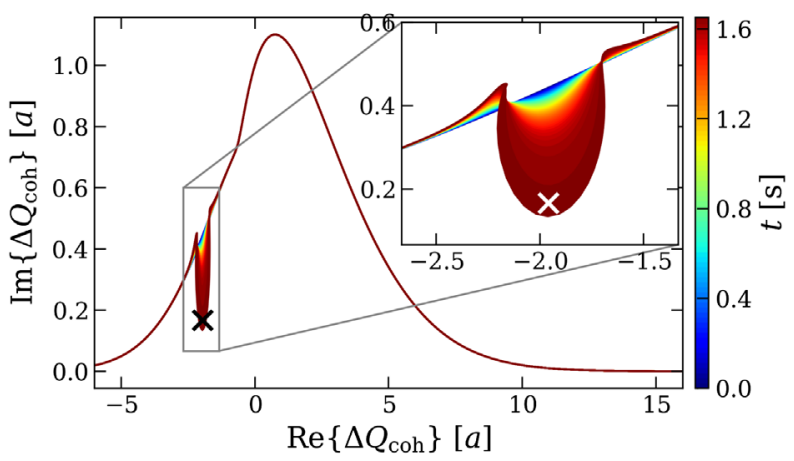

(b)

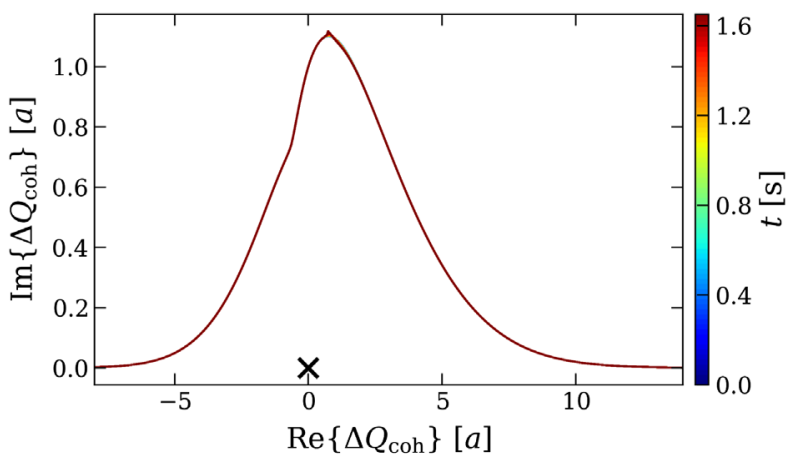

(c)

FIG. 8. Change of distribution in (a) due to diffusion driven by horizontal wakefields, and corresponding evolution of the horizontal and vertical stability diagrams in (b) and (c), respectively. The dashed line in (a) marks where $Q_{x}\left(J_{x}, J_{y}\right)=Q_{\mathrm{LD} x}$. The cross at $\Delta Q_{\text {coh }}=-1.47 \times 10^{-4}+1.25 \times 10^{-5} i$ in (b) marks the tune of the least stable mode in the horizontal plane. No mode, nor noise, is included in the vertical plane.

flattening causes a change of the horizontal stability diagram that is illustrated in Fig. 8(b). A borehole is drilled in the stability diagram directly at the frequency of the least stable mode. The drilling speeds up as the instability is approached, since $\operatorname{Im}\left\{Q_{\mathrm{LD} x}\right\}$ approaches 0 . The vertical stability diagram in Fig. 8(c) is barely modified in this process. 


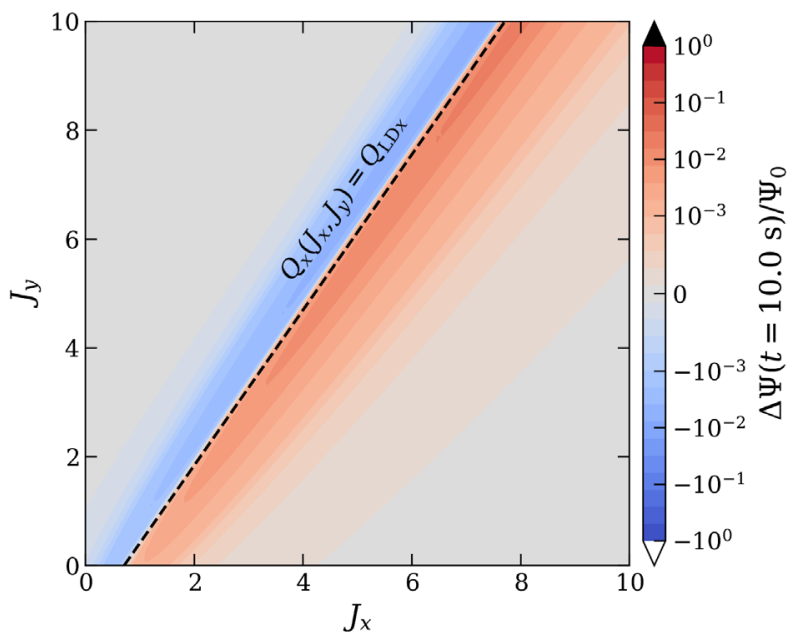

(a)

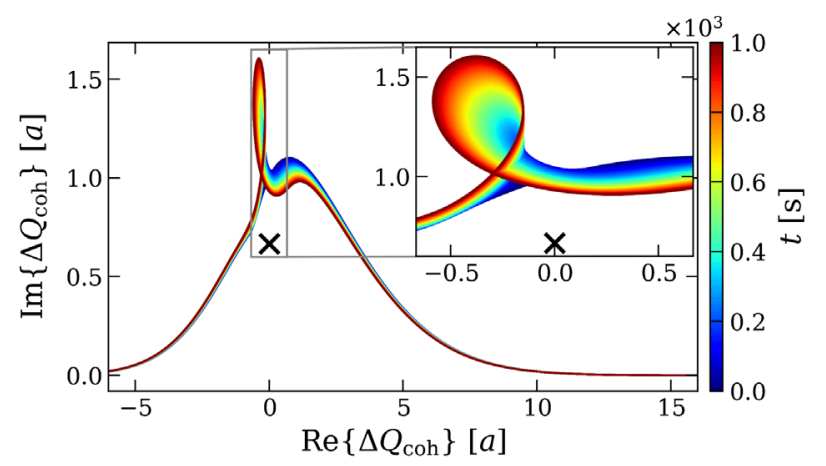

(b)

FIG. 9. Change of distribution in (a) and evolution of the horizontal stability diagram in (b), due to diffusion driven by horizontal wakefields. The dashed line in (a) marks the actions where $Q_{x}\left(J_{x}, J_{y}\right)=Q_{\mathrm{LD} x}$. The cross at $\Delta Q_{\mathrm{coh}}=5.00 \times 10^{-5} i$ in (b) marks the tune of the least stable mode.

In the second case, the least stable mode is at $\Delta Q_{\text {coh }}=5.00 \times 10^{-5} i$. The relative change of the distribution after $10 \mathrm{~s}$ and the evolution of stability diagram are illustrated in Fig. 9. The distribution changes as in the first case, with the main difference being a shift of the resonant tune, due to the difference in $\operatorname{Re}\left\{\Delta Q_{\text {coh }}\right\}$. Instead of the drilling of a borehole in the stability diagram, the stability threshold does initially grow, before a loop starts developing. This loop is difficult to interpret and it causes challenges for the numerical algorithm used to calculate $\alpha$ and $\Delta Q_{\mathrm{LD} x}$. Therefore, the diffusion coefficient has, in the calculation presented here, been kept constant at the initial value. Similar evolutions have been calculated with time evolving diffusion coefficient. With zero real tune shift, it was derived in Sec. II F that a local flattening at the resonant actions would initially lead to an increased stability threshold, which has been verified.

The evolution of $\operatorname{Im}\left\{\Delta Q_{\mathrm{LD} x}\right\}$ for these two cases is presented in Fig. 10. In the derivation of the analytical latency in Sec. II F, it was assumed that $\alpha$ would stay constant

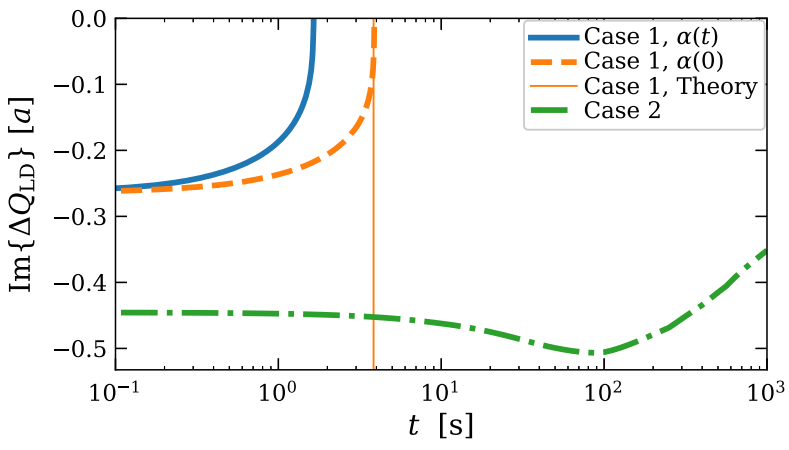

FIG. 10. Evolution of $\operatorname{Im}\left\{\Delta Q_{\mathrm{LD}}\right\}$ for two test cases illustrated in Figs. 8 and 9. The vertical line marks the latency estimated with Eq. (50) for the calculation represented by the dashed line (“Case 1, $\alpha(0)$ ").

at its initial value $\alpha(0)$. This is not correct. As the borehole is drilled, the curvature of the stability diagram will increase, leading to a reduction of $\alpha(t)$ with time. In addition to the calculations presented in Figs. 8 and 9, an additional calculation has been executed of case 1 where $\alpha$ has artificially been kept constant at its initial value. The latencies for case 1 in the PyRADISE calculations are $1.65 \mathrm{~s}$ with evolving $\alpha(t)$ and $3.88 \mathrm{~s}$ with constant $\alpha(0)$, in comparison to $3.84 \mathrm{~s}$ estimated with the analytical theory in Eq. (50). Note also that even if the stability margin initially increases for case 2 with $\operatorname{Re}\left\{\Delta Q_{\text {coh }}\right\}=0$, it eventually decreases. Nevertheless, the latency is longer than the $87.0 \mathrm{~s}$ that would have been wrongfully estimated with Eq. (50), which assumed $\left|\operatorname{Re}\left\{\Delta Q_{\text {coh }}\right\} / \operatorname{Im}\left\{\Delta Q_{\text {coh }}\right\}\right| \gg 1$.

\section{B. Detuning margin}

Test case 1 in Sec. VA is representative of the type of modes that typically are the least stable modes in the LHC. The drilling of a borehole in the stability diagram can be expected, and this drilling speed increases as the instability is approached. The latency for the least stable mode $\Delta Q_{\text {coh }}=-1.47 \times 10^{-4}+1.25 \times 10^{-5} i$, with different detuning margins, is presented in Fig. 11. By scaling the detuning margin by 1 order of magnitude, from $10 \%$ to $100 \%$, the latency increases by more than 4 orders of magnitude.

Note the three different sets of points calculated with PyRADISE: (i) For the points labeled " $\alpha(0)$ ", the factor $\alpha$ has been kept constant at its initial value, as was assumed in the analytical latency in Eq. (50). The agreement between these points and the approximative analytical latency is striking for $a<2 a_{\text {thresh }}$; (ii) The points labeled " $\alpha(t)$ " are considered to best represent the physics at hand, self-consistently solving for both $\alpha$ and the diffusion coefficient as the distribution changes. However, for large stability margins, $a>2 a_{\text {thresh }}$, the linear extrapolation of the damped mode becomes increasingly inaccurate at $t=0$. Furthermore, as the borehole is drilled this deep for this mode, $\alpha=\partial M_{\mathrm{LD}} / \partial M_{\text {coh }}$ is quickly varying due to an increased 


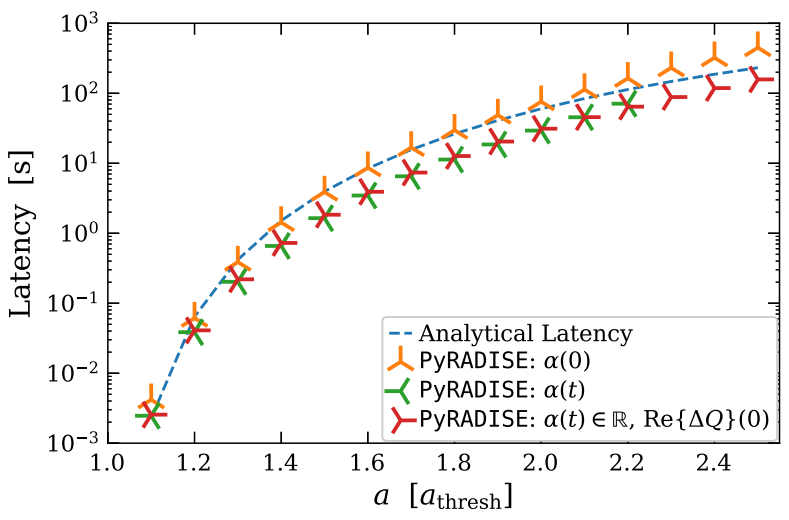

FIG. 11. Latency for a mode of coherent tune $\Delta Q_{\text {coh }}=-1.47 \times 10^{-4}+1.25 \times 10^{-5} i$, stabilized by octupole detuning with $a_{\text {thresh }}=5 \times 10^{-5}$ and $b=-0.7 a$.

curvature of the stability diagram. Thus, a small inaccuracy in the initial $\operatorname{Re}\left\{\Delta Q_{\mathrm{LD}}\right\}$ will cause an unphysical drift of $\operatorname{Re}\left\{M_{\mathrm{LD}}\right\}$ by use of Eq. (26) for $\left|M_{\text {coh }}\right| \gg \epsilon$. This can prevent the instability in the numerical calculation, by nonphysically varying the resonant action; (iii) Therefore, for the points labeled " $\alpha(t) \in \mathbb{R}, \operatorname{Re}\{\Delta Q\}(0)$ ", the real tune of the damped mode has been forced constant at the initial value, while $\alpha$ has been allowed to evolve in time, but forced to be real. This is considered the best approximation of the drilling at large detuning margins for this mode.

\section{Experiment in the LHC}

An experiment has been conducted in the LHC, where up to 13 proton bunches per beam, with a minimal separation of $5.25 \mu \mathrm{s}$, were accelerated to flat top under nominal conditions $[6,7]$. With this injection scheme, all beam-beam interactions were avoided. While at flat top, subsets of the bunches were excited by a controllable noise source, acting equally on all particles per bunch per turn with effectively a white spectrum over multiple turns. The time from the noise was turned on until the bunches went unstable was measured as the instability latency. Only latencies between $1 \mathrm{~min}$ and $60 \mathrm{~min}$ were acceptable, due to constraints of the theory and the allotted machine time. A few key parameters are listed in Table I. The condition for diffusion in Eq. (53) has been found to be met as $R_{D} \gtrsim 200$ for all investigated bunches.

The experiment was conducted at flat top with $\beta^{*}=1 \mathrm{~m}$. For the corresponding optical functions, the linear detuning coefficients in the horizontal plane can be calculated for beam 1 (B1) as

$$
\begin{aligned}
& a_{x}=+543.2 \cdot I_{\mathrm{oct}} \cdot \frac{\varepsilon_{n x 0}}{\beta_{\mathrm{rel}} \gamma_{\mathrm{rel}}}, \\
& b_{x}=-383.6 \cdot I_{\mathrm{oct}} \cdot \frac{\varepsilon_{n y 0}}{\beta_{\mathrm{rel}} \gamma_{\mathrm{rel}}},
\end{aligned}
$$

where $I_{\text {oct }}$ is the octupole current, limited to a maximum of about $\pm 570 \mathrm{~A}, \varepsilon_{n j 0}$ is the initial normalized emittance in
TABLE I. Important parameters during a latency experiment conducted in the LHC [6,7]. The bunch parameters are averaged values in, e.g., the horizontal plane of beam $1(\mathrm{~B} 1 \mathrm{H})$.

\begin{tabular}{lccc}
\hline \hline Parameter & Unit & Fill 1 & Fills 2 \& 3 \\
\hline Energy per proton & {$[\mathrm{TeV}]$} & 6.5 & 6.5 \\
Horizontal tune, $Q_{x}$ & {$[\mathrm{mod} 1]$} & 0.275 & 0.275 \\
Vertical tune, $Q_{y}$ & {$[\bmod 1]$} & 0.295 & 0.295 \\
Synchrotron tune, $Q_{s}$ & {$[1]$} & 0.00191 & 0.00191 \\
Revolution frequency, $f_{\text {rev }}$ & {$[\mathrm{kHz}]$} & 11.2455 & 11.2455 \\
Total RF voltage & {$[\mathrm{MV}]$} & 12 & 12 \\
Normalized emittance B1H & {$[\mu \mathrm{m}]$} & 1.86 & 1.91 \\
Normalized emittance $\mathrm{B} 1 \mathrm{~V}^{\mathrm{a}}$ & {$[\mu \mathrm{m}]$} & 1.14 & 1.18 \\
Normalized emittance B2H & {$[\mu \mathrm{m}]$} & 0.87 & 1.37 \\
Normalized emittance B2 $\mathrm{V}^{\mathrm{a}}$ & {$[\mu \mathrm{m}]$} & 1.48 & 1.24 \\
Intensity & {$\left[10^{11} \mathrm{p} / \mathrm{b}\right]$} & 0.91 & 1.10 \\
Bunch length, $4 \sigma_{s}$ & {$[\mathrm{~ns}]$} & 1.12 & 1.07 \\
\hline \hline
\end{tabular}

${ }^{\mathrm{a}}$ Measured with the beam synchrotron radiation telescope.

plane $j$, and $\beta_{\text {rel }}$ and $\gamma_{\text {rel }}$ are the relativistic factors. For beam 2 (B2), the prefactors are slightly changed to 531.3 and -383.4 .

The latency for a specific bunch depends strongly on the individual bunch parameters, especially the emittance and intensity. This can mask the dependence on the machine parameters. The analytical latency for the worst mode, given by Eq. (50), is illustrated as a function of the chromaticity and feedback gain in Fig. 12(a). The second shortest latency is given in Fig. 12(b), in units of the shortest latency, hinting at whether a single mode is sufficient in modeling the latency or not. Latencies below $0.6 \mathrm{~s}$ have been marked as if the bunch was already unstable. Note that the predicted latency varies over more than 6 orders of magnitude in this limited parameter space, where neither the octupole current, nor the noise amplitude, nor the bunch specific parameters have been varied.

The latencies of 8 bunches in B2 in fill 1 have been investigated in more detail, including the bunch specific parameters, such as the intensity, emittances, bunch length, and applied noise amplitude. The comparison between the experimental latencies, and the latencies calculated with PyRADISE is given in Fig. 13(a). The first set of bunches (450-1350) was the first experimental realization of this mechanism. These bunches were acted on by external noise in two intervals, and the emittance measurement was by chance sub-par, detailed below, giving cause for the large error bars. They went unstable with $a \approx 2 a_{\text {thresh }}$, assuming a Gaussian bunch. The second set of bunches (1950-2850) was acted on in slightly better conditions, allowing for a better quantitative comparison to the diffusion model. The first three of these bunches went unstable with $a \approx 2.4 a_{\text {thresh }}$, assuming a Gaussian bunch. Bunch 2850 did not go unstable during the experiment. The chromaticity was $Q^{\prime}=15$, and the damping time due to the feedback was 170 turns. For this configuration, all modes with positive $\operatorname{Im}\left\{\Delta Q_{\text {coh }}\right\}$, and centered maximally 


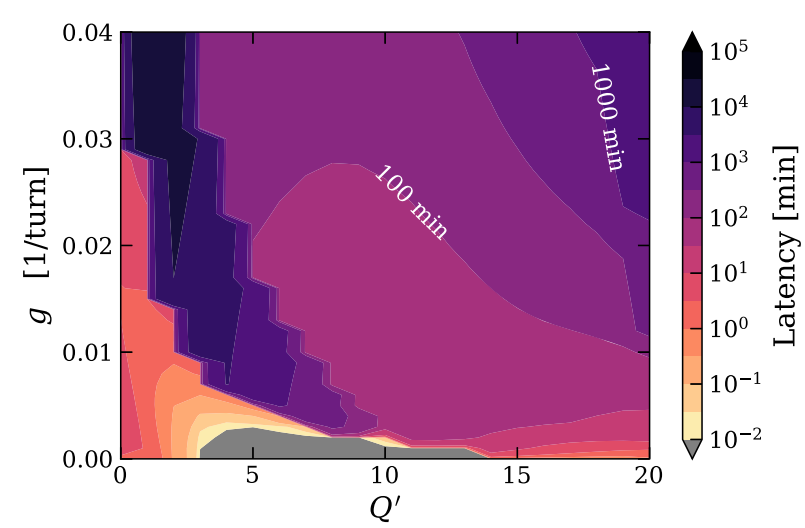

(a)

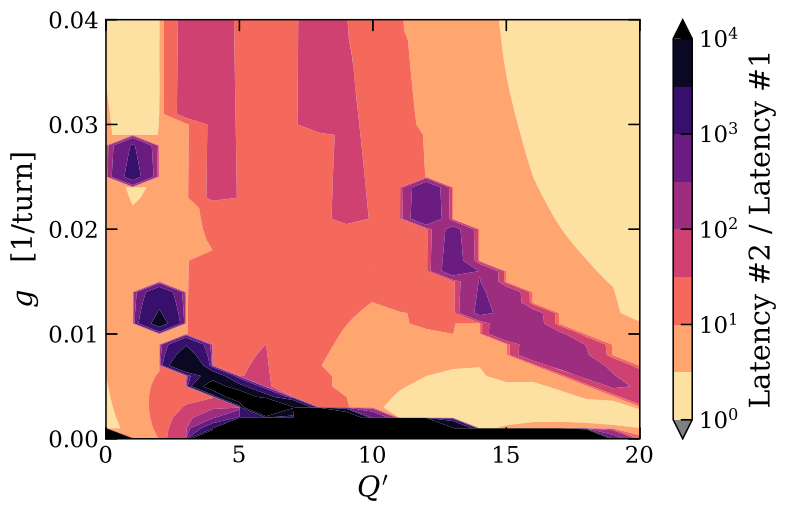

(b)

FIG. 12. Analytical latency of the worst horizontal mode in B1 in the LHC in (a) and second worst mode in (b), for the parameter values: normalized emittance $\varepsilon_{j}=1.4 \mu \mathrm{m}$; bunch length $4 \sigma_{s}=1.05 \mathrm{~ns}$; intensity $N=1.1 \times 10^{11} \mathrm{p} / \mathrm{b}$; noise amplitude $\sigma_{\text {ext }}=1 \times 10^{-4}$; octupole current $I_{\text {oct }}=400 \mathrm{~A}$. The feedback gain $g$ is defined for a system featuring a single kicker, such that the corresponding damping time in number of turns is $2 / \mathrm{g}$.

10 sidebands from the bare tune, are displayed in Fig. 13(b). Since one mode stands out, a single mode should represent the diffusion well.

The error bars in Fig. 13(a) are large, because the latency scales quadratically or faster with a set of uncertain parameters, as given by Eq. (52). The considered most significant uncertainties are: (i) The emittance measured with the beam synchrotron radiation telescope (BSRT) was in 2018 found to have a $10 \%$ accuracy [33]. During the measurement of bunches 450-1350, the emittance measurement of all bunches displayed an unphysical oscillatory drift. The total uncertainty in the emittance of these bunches has therefore been estimated to $25 \%$. As the linear detuning coefficients in Eq. (56) are proportional to the emittance, this uncertainty affects the knowledge of the stability margin. Furthermore, the horizontal noise $\sigma_{\text {ext }}$ is given in units of beam divergence, such that $\sigma_{\mathrm{ext}}^{2} \varepsilon_{x}$ is constant. Thus, the larger emittance corresponds to the upper error bar on the latency. A $10 \%$ uncertainty in the emittance causes an uncertainty of a factor $\sim 2$ on the latency for these detuning margins. It was

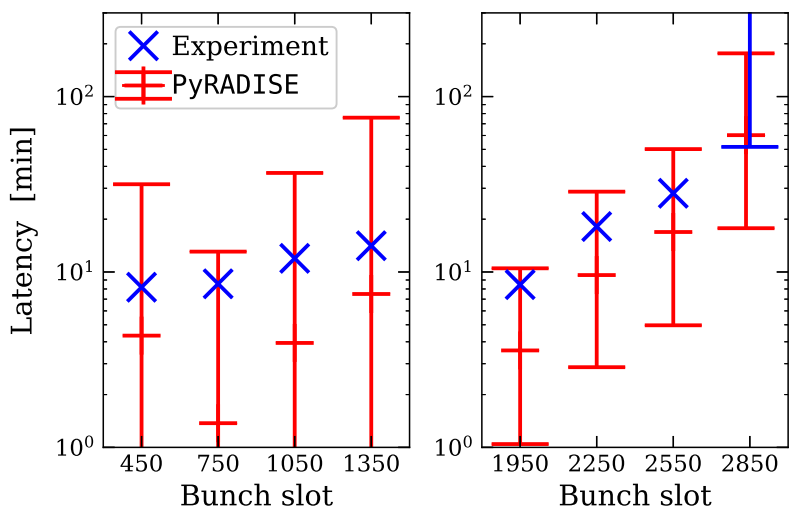

(a)

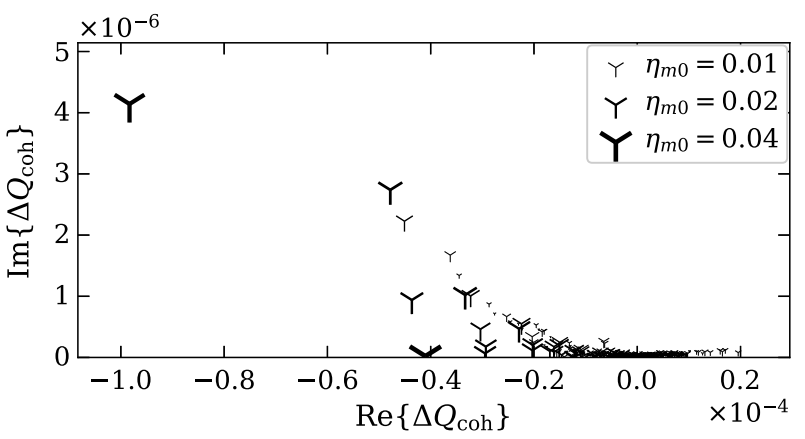

(b)

FIG. 13. Comparison between experiment in the LHC [6,7] and numerical latency found by PyRADISE solving the diffusion equation introduced in this paper in (a), for the worst mode found by BimBim in (b). The error bars in (a) are due to uncertain measurements of the emittances, chromaticity, feedback gain, and external noise amplitude during the experiment. Predicted latencies below $1 \mathrm{~min}$ are not shown, as the dynamics require more time to be modeled accurately as a diffusion (see Sec. IV C). Bunch 2850 did not go unstable before $I_{\text {oct }}$ was reduced after $52 \mathrm{~min}$.

found in 2018 that the BSRT on average underestimated the emittance, favoring the upper error bar [34]; (ii) The noise amplitude was experimentally known with low accuracy. By comparing the emittance growth of these bunches in B2 with macroparticle simulations, the noise amplitude has been scaled by a factor $3.85 \pm 0.30$ to $\sigma_{\text {ext }} \in[3.4,14.3] \times 10^{-4}$ in units of the beam divergence, ignoring the uncertainty; (iii) The gain is considered to be estimated with approximately $10 \%$ margin; (iv) The chromaticity was not measured on the day of the fill presented in Fig. 13. It is therefore only estimated with accuracy \pm 1 . The uncertainty in the feedback gain and chromaticity causes an uncertainty in $\Delta Q_{\text {coh }}$ and $\eta_{m 0}$. The shortest and longest latency has been calculated with $Q^{\prime}=15 \pm 1$ and $g=(12 \pm 1) \times 10^{-3}$. The uncertainty in gain and chromaticity only causes a small part of the total error bar on the latency, in comparison to that caused by the uncertainty in the emittance.

The experiment was repeated in similar conditions, but with the chromaticity set to $Q^{\prime}=5$. No instabilities were 
reached during the experiment at the initial octupole current. Furthermore, by reducing $I_{\text {oct }}$, it was found that the stability threshold had barely been modified by the noise. This is in qualitative agreement with the predictions in Fig. 12(a), where the latency is more than an order of magnitude longer for $g=0.01$ and $Q^{\prime} \approx 5$, than for $g=0.01$ and $Q^{\prime} \approx 15$. However, other instability mechanisms have been observed with low positive chromaticities [35]. They may limit the range of acceptable chromaticities for the operation of the LHC.

\section{Physics fills in the LHC}

This study was mainly motivated by the observations of instabilities developing when the beam was maintained in a steady configuration for a few to tens of minutes, with an octupole current less than twice the required value expected from linear Vlasov theory, with a Gaussian distribution. Consequently, the LHC was operated with at least twice as much octupole current as initially expected to mitigate this instability $[2,36]$. The detuning coefficients in normal operation in 2018 were on average close to the ones in the experiment described in the previous section, with an average normalized emittance of $1.9 \mu \mathrm{m}$ and octupole current $I_{\text {oct }}=280$ A. The noise was measured at approximatively $\sigma_{\text {ext }}=6 \times 10^{-5}$, with the operational gain of $g=0.01$ [25]. The predicted latency for the average bunch at $Q^{\prime}=15$ and $g=0.01$ is $120 \mathrm{~min}$, which is slightly larger than the value in Fig. 12, due to the lower noise amplitude. With a $15 \%$ lower emittance, the expected latency drops to $9.9 \mathrm{~min}$, illustrating again how sensitive the latency is to the individual bunch parameters. Similar reductions of the latency occur from an equal reduction of $I_{\text {oct }}$ or increase of the intensity. We note that the latencies obtained with the formula in Eq. (50) are usually overestimated with respect to the numerical estimates. Nevertheless, such latencies seem compatible with safe operation of the LHC. With this study we therefore confirm the observed efficiency of the mitigation strategy of operating with a margin in the octupole current, initially implemented based on empirical evidence only.

\section{DISCUSSION}

The analytical latency and simulated results that has been presented, assumed that a single mode is dominant. The ratio of the second shortest latency in B1 in the LHC to the shortest latency was presented in Fig. 12(b). In the configurations that has been studied, the shortest latency is at least an order of magnitude shorter than the second shortest. In configurations where two modes are relevant, one of three things can happen: (i) The modes flatten the distribution at exactly the same frequency, reducing the latency by maximally a factor 2 for uncoupled modes; (ii) The modes flatten the distribution at close but different frequencies, widening and increasing the diffusion, causing either an increase or decrease of the latency, depending on the separation in frequency; (iii) The modes flatten the distribution at well separated frequencies, with no impact on one another. A case by case study would be required for exact predictions of the impact of the second worst mode.

In this paper, only the wake driven diffusion has been considered. It is found to be a strong candidate for the driver of the instabilities of long latencies observed in the LHC. Diffusion that can counteract the drilling in the stability diagram has not been studied in detail here. The diffusion due to the first term on the rhs of Eq. (33) and due to intra-beam scattering (IBS) were studied in [37], and found to only weakly increase the latency in an LHC-like configuration. The effect of IBS will depend on the ratio between the latency and the IBS driven emittance doubling time, which is in the order of days in the LHC at flattop. If the latency, ignoring the IBS, is similar to the doubling time, it may be increased indefinitely. However, in such configurations, the latent instability will not be a problem for operation of the LHC.

\section{CONCLUSION}

Transverse instabilities with latencies from a few to tens of minutes have been observed in the LHC, both in regular operation and in dedicated experiments. In this paper, we have considered the hypothesis that such instabilities are due to a long-term evolution of the transverse distribution, which leads to a loss of Landau damping. The mechanism that has been studied in detail here, is that external sources of noise excite the beam, which then acts back on itself through electromagnetic wakefields. The coherent response of the beam has been modeled as a set of damped harmonic oscillators, modeling the least stable wakefield driven modes. The impact of the wakefields on the individual particles has been modeled as a diffusion, which is narrow in frequency space around the real frequency of the least stable mode. Thus, the diffusion is also narrow in action space around the resonant action, causing a local flattening of the distribution in 2D action space. Numerical calculations with PyRADISE have shown that the distribution evolution efficiently drills a borehole in the stability diagram at the frequency of the least stable mode, if the absolute value of the real tune shift of the coherent mode is large compared to the imaginary part. This can cause an instability with a latency. One of the most effective techniques to extend the latency, and thus mitigate this instability mechanism, is to operate with a large stability margin. An analytical expression for the latency has been derived in Eq. (50) under strict assumptions, and found to consistently be a factor $\sim 2$ longer than the latency found with PyRADISE. The predicted latency is sensitive to the input, being proportional to the second or higher power of multiple parameters. Consequently, the latencies of the configurations considered in this paper vary by more than 8 orders of magnitude.

The new theory and numerical solver have been compared with latency experiments performed in the 
LHC [6,7]. The latencies measured in the experiment have been reproduced, albeit with large error bars, eventually causing instabilities with more than twice the required detuning strength for a Gaussian distribution. The latencies in regular LHC operation are also in agreement with the new theory. Thus, it can be concluded that the loss of Landau damping observed in the LHC is most probably caused by noise excited wakefields driving a diffusion of individual particles. One important result is the confirmation that the latency is approximately an order of magnitude longer at an intermediate chromaticity $Q^{\prime} \approx 5$ with a feedback driven damping time of 200 turns, than at $Q^{\prime} \approx 15$. This constitutes an alternative working point for the LHC worth considering in view of relaxing intensity limits that may arise due to this mechanism.

Going forward, there are various aspects of this mechanism that deserve further studies: (i) Improve the modeling of the beam response, compared to the current underdamped stochastic harmonic oscillator with frequency found by a linear extrapolation; (ii) Study the impact of crab-cavity amplitude noise, which should give a large noise moment $\eta_{m 1}$ to headtail modes; (iii) Perform experiments showing the qualitative effect of the diffusion. The local flattening in 2D action space will not be resolvable with beam profile measurements, but the drilling of a borehole in the stability diagram can be measured through beam transfer function measurements.

\section{ACKNOWLEDGMENTS}

The authors would like to thank: Nicolas Mounet for several discussions, both on the dynamics of modes residing inside the stability diagram and on the wake force; Armando Bazzani for discussions on the action-dependent diffusion; Rogelio Tomás for discussions on the harmonic excitation with frequency inside the incoherent spectrum; Valeri Lebedev for motivating discussions; and Elias Métral and Gianluigi Arduini for constructive feedback on this manuscript. This research was supported by the HL-LHC project.

\section{APPENDIX: HARMONICALLY DRIVEN DISTRIBUTION EVOLUTION}

The body of this paper models damped, wake-driven modes as under-damped stochastically driven harmonic oscillators. Due to their stochastic nature, the resulting force is modeled as a diffusion. However, in the limit $\operatorname{Im}\left\{\Delta \Omega_{m}\right\} \rightarrow 0$, the force becomes a pure harmonic excitation. In this appendix, we study the resulting distribution dynamics when a bunch is affected by a harmonic excitation.

Consider that a harmonic oscillator of angular frequency $\omega$ is harmonically driven by a real impulse $P_{h} \cos \left(\omega_{h} t\right)$, kicked once per turn, changing the one-turn Hamiltonian in Eq. (3) to

$$
\mathcal{H}=\mathcal{H}_{0}-\sqrt{2 J} \cos (\phi) f_{\text {rev }} P_{h} \cos \left(\omega_{h} t\right),
$$

where $\mathcal{H}_{0}$ models the free motion. In the simple case of no amplitude detuning, $\mathcal{H}_{0}=\omega J$, the motion of the harmonic oscillator will consist of the free and the forced motion, as due to an ac dipole [38]. The amplitude of the forced motion is

$$
A(\omega)=\frac{\omega f_{\mathrm{rev}} P_{h}}{\left|\omega^{2}-\omega_{h}^{2}\right|}
$$

which is singular for a harmonic oscillator at exactly the driving frequency.

In an accelerator, the incoherent particle frequency is typically amplitude dependent, for instance due to Landau octupoles as given by Eq. (9). Therefore, a particle's amplitude will no longer be singular, because the resonance condition is disrupted as the amplitude grows. For a harmonic excitation in only one plane, only the amplitude detuning in that plane requires consideration. Hamilton's equations of motion for $\phi$ and $J$ then reads $[10,39]$

$$
\begin{gathered}
\mathcal{H}=\omega_{0} J+\frac{\tilde{a}}{2} J^{2}-\sqrt{2 J} \cos (\phi) f_{\mathrm{rev}} P_{h} \cos \left(\omega_{h} t\right), \\
\dot{\phi}=\omega_{0}+\tilde{a} J-\frac{f_{\mathrm{rev}} P_{h}}{\sqrt{2 J}} \cos (\phi) \cos \left(\omega_{h} t\right) \\
=\omega_{0}+\tilde{a} J-\frac{f_{\text {rev }} P_{h}}{2 \sqrt{2 J}}\left[\cos \left(\phi+\omega_{h} t\right)+\cos \left(\phi-\omega_{h} t\right)\right] \\
\dot{J}=-\sqrt{2 J} \sin (\phi) f_{\text {rev }} P_{h} \cos \left(\omega_{h} t\right) \\
=-\sqrt{\frac{J}{2}} f_{\text {rev }} P_{h}\left[\sin \left(\phi+\omega_{h} t\right)+\sin \left(\phi-\omega_{h} t\right)\right] .
\end{gathered}
$$

The harmonic frequency corresponds to a harmonic action $J_{h}$ such that $\dot{\phi}\left(J_{h}\right)=\omega_{h}$. One can find new conserved properties of the full Hamiltonian [39], but that is not the current goal. Here, we will study the motion of particles starting at the harmonic action $J_{h}$ and any initial phase $\phi_{0}$ in terms of the original action corresponding to $\mathcal{H}_{0}$. The goal is to get an expression for the width of the action oscillation, and the period of this motion. The terms with the high angular frequency $\dot{\phi}+\omega_{h}$ will not produce a macroscopic change of action. The terms with the potentially low frequency $\dot{\phi}-\omega_{h}$ will have the same sign for an extended period of time, and can cause a macroscopic change of action for particles close to the harmonic action $J_{h}$. The beating excitation leads to a slowly oscillating action evolution of a certain width and period, which will be estimated in the following by directly solving Eqs. (A4) and (A5) in multiple limits. 
In the limit of large harmonic excitation, $f_{\text {rev }} P_{h} / \sqrt{J} \gg \tilde{a}\left(J-J_{h}\right)$, and neglecting the highfrequency term, the equations of motion become

$$
\begin{aligned}
& \dot{\phi}=\omega_{h}-\frac{f_{\text {rev }} P_{h}}{2 \sqrt{2 J}} \cos \left(\phi-\omega_{h} t\right), \\
& \dot{J}=-\sqrt{\frac{J}{2}} f_{\text {rev }} P_{h} \sin \left(\phi-\omega_{h} t\right) .
\end{aligned}
$$

Regardless of the initial phase $\phi_{0}$, the phase will approach its one stable value at $\phi-\omega_{h} t=-\pi / 2+2 \pi n$, where $n$ is an integer. The corresponding time derivative of the action is positive. The action will grow until $f_{\text {rev }} P_{h} / \sqrt{J} \sim \tilde{a}\left(J-J_{h}\right)$.

Alternatively, if the harmonic excitation could be treated as a weak perturbation, $f_{\text {rev }} P_{h} / \sqrt{J} \ll \tilde{a}\left(J-J_{h}\right)$, the last term in Eq. (A4) can be neglected. If one also assumes small phase offsets $\left|\phi-\omega_{h} t\right| \ll 1$ and small action offsets $\left|J-J_{h}\right| \ll J_{h}$, the equations of motion read

$$
\begin{gathered}
\dot{\phi}=\omega_{h}+\tilde{a}\left(J-J_{h}\right), \\
\dot{J}=-\sqrt{\frac{J_{h}}{2}} f_{\text {rev }} P_{h}\left(\phi-\omega_{h} t\right) .
\end{gathered}
$$

These are the equations of motion of a simple harmonic oscillator, but it is the phase and action that is oscillating. In the following it is assumed for simplicity, but without loss of generality, that $\tilde{a}>0$ and that $\phi_{0} \in(-\pi, \pi)$. It can be verified by insertion that the equations of motion, combined with the initial conditions $J(0)=J_{h}$ and $\phi(0)=\phi_{0}$, are solved by

$$
\begin{gathered}
\phi(t)=\omega_{h} t+\phi_{0} \cos \left(2 \pi \frac{t}{\tau_{h \min }}\right), \\
J(t)=J_{h}-W_{h \min } \sin \left(2 \pi \frac{t}{\tau_{h \min }}\right),
\end{gathered}
$$

where the minimal period $\tau_{h \text { min }}$ and action offset amplitude $W_{h \text { min }}$ are given by

$$
\begin{gathered}
\left(\tau_{h \min } f_{\text {rev }}\right)^{2}=\frac{(2 \pi)^{2}}{\sqrt{2 J_{h}} P_{h} a \pi}, \\
W_{h \min }\left(\phi_{0}\right)=\frac{\left(2 J_{h}\right)^{1 / 4} \sqrt{P_{h} \pi}}{2 \sqrt{a}} \frac{\phi_{0}}{\pi},
\end{gathered}
$$

where the detuning coefficients have been rewritten with $\tilde{a}=\omega_{\text {rev }} a$. The initial phase is the maximum phase offset, fulfilling the assumption of small $\left|\phi-\omega_{h} t\right|$. The maximum action offset $\left|J-J_{h}\right|$ is small if the harmonic excitation is weak. The action offset amplitude is proportional to $\phi_{0}$, which is assumed small.
The maximum action offset should occur for large initial phases $\left|\phi_{0}\right| \rightarrow \pi^{-}$, for which the approximation $\sin \left(\phi_{0}\right)=\phi_{0}$ in Eq. (A9) is wrong. In this case, both $\dot{\phi}$ and $\dot{J}$ approaches 0 initially and there is almost an asymptotic motion with an infinite period $\tau_{h} \rightarrow \infty$. This will, however, be prevented by the last term in Eq. (A4). The action offset amplitude will, on the other hand, not grow infinitely. Since $\left|\sin \left(\phi-\omega_{h} t\right)\right| \leq\left|\phi-\omega_{h} t\right|$, the maximum width in action can be approximated by $\left|W_{h \min }(\pi) 2 / \pi\right|$ as

$$
W_{h}=\frac{\left(2 J_{h}\right)^{1 / 4} \sqrt{P_{h}}}{\sqrt{a \pi}} .
$$

Due to different harmonic periods of different particles, the corresponding distribution oscillation will decohere with time.

To test the analytical derivations, numerical tracking simulations have been run with $a=5 \times 10^{-3}$ for particles starting at the harmonic action $J_{h}=2$ with phases $\phi_{0}$ uniformly distributed on $[-\pi, \pi]$. The peak-to-peak action variation divided by two and the period of the oscillation is presented in Fig. 14. At small $\left|\phi_{0}\right|$, the expressions in Eqs. (A12) and (A13) are shown to be correct. At large $\left|\phi_{0}\right|$, the action offset amplitude approaches $W_{h}$ given by Eq. (A14), while the period grows substantially. For large $P_{h} / a J_{h}$, the motion is different, but qualitatively similar. Note that the largest widths clearly breaks the assumption $W_{h} \ll J_{h}=2$. For $a<0$, the general picture is the same, but the particles oscillate around $\phi-\omega_{h} t=\pi$ instead of 0 .

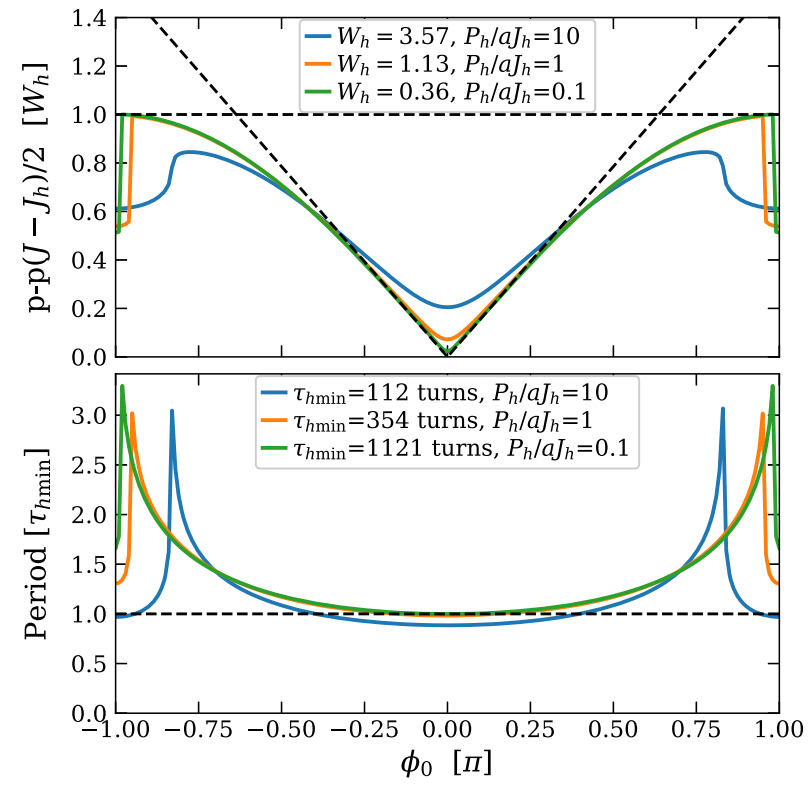

FIG. 14. Peak-to-peak (p-p) action variation amplitude on the top, and period on the bottom, for particles starting at $J_{h}=2$ with various initial phases $\phi_{0}$. The dashed diagonal and horizontal lines on the top correspond to Eq. (A13) and Eq. (A14), respectively. The dashed horizontal line on the bottom corresponds to Eq. (A12). 


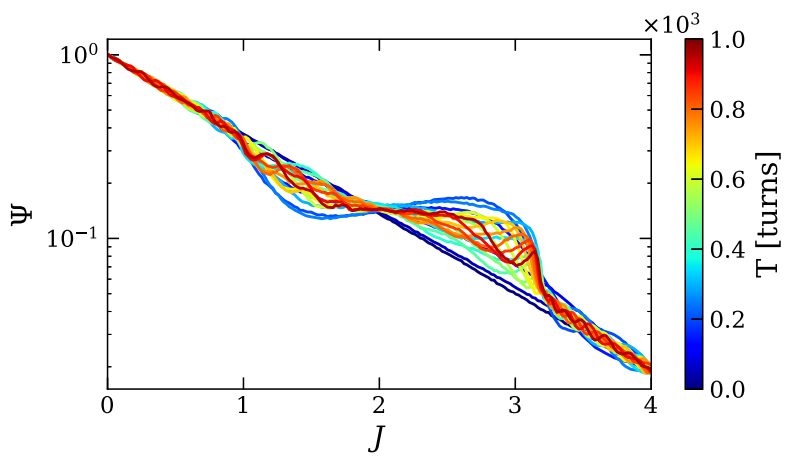

(a)

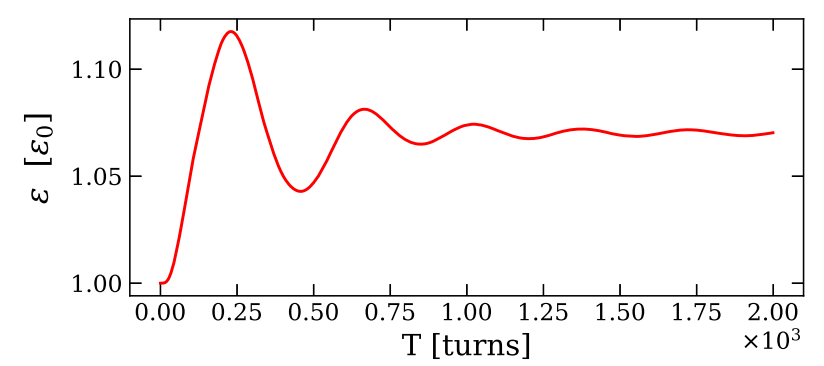

(b)

FIG. 15. Evolution of the distribution every 50 turns in (a) and emittance in (b) of a bunch that is driven by a harmonic force $P_{h} \cos \left(\omega_{h} t\right)$. The in-plane detuning coefficient is $a=5 \times 10^{-3}$, corresponding to a detuning of $a J$.

To study how the harmonic perturbation affects the bunch distribution, a macroparticle simulation has been run with $P_{h}=10^{-2}, a=5 \times 10^{-3}$, and $J_{h}=2$, equal to the curve labeled $P_{h} / a J_{h}=1$ in Fig. 14. The bunch evolution is presented in Fig. 15. The action oscillation around $J_{h}=2$ is clear. The full width is close to $2 W_{h}=2.2$, given by Eq. (A14). For this large $W_{h} / J_{h}$, the amplitude is not symmetric around $J_{h}$, but slightly larger for $J>J_{h}$ than for $J<J_{h}$. The emittance period is as expected longer than the minimum incoherent action period, $\tau_{h \min }=354$ turns, given by Eq. (A12). This process cannot be modeled as a diffusion because it is deterministic and not stochastic. It may, however, enhance the diffusion across $J_{h}$ driven by other stochastic processes.

[1] J. S. Berg and F. Ruggiero, Landau damping with twodimensional betatron tune spread, CERN, Geneva, Switzerland, Report No. CERN-SL-AP-96-071-AP, 1996.

[2] X. Buffat, G. Arduini, D. Amorim, S. Antipov, N. Biancacci, L. Carver, S. V. Furuseth, G. Iadarola, K. Li, L. Mether, E. Métral, N. Mounet, A. Oeftiger, A. Romano, G. Rumolo, B. Salvant, M. Schenk, T. Pieloni, and C. Tambasco, Transverse instabilities, in Proceedings of the 9th Evian Workshop on LHC Beam Operation, Evian-lesBains, France, Rep. CERN-ACC-2019-059, Jan. 2019.

[3] E. H. Maclean, R. Tomás, F. S. Carlier, M. S. Camillocci, J. W. Dilly, J. C. de Portugal, E. Fol, K. Fuchsberger,
A. G.-T. Valdivieso, M. Giovannozzi, M. Hofer, L. Malina, T. H. B. Persson, P. K. Skowronski, and A. Wegscheider, New approach to LHC optics commissioning for the nonlinear era, Phys. Rev. Accel. Beams 22, 061004 (2019).

[4] L. R. Carver, X. Buffat, K. Li, E. Métral, and M. Schenk, Transverse beam instabilities in the presence of linear coupling in the Large Hadron Collider, Phys. Rev. Accel. Beams 21, 044401 (2018).

[5] D. Amorim, Study of the transverse mode coupling instability in the CERN Large Hadron Collider, Ph.D. thesis, Université Grenoble Alpes, Grenoble, France, 2019, CERN-THESIS-2019-272.

[6] S. V. Furuseth, D. Amorim, S. A. Antipov, X. Buffat, N. Mounet, E. Métral, T. Pieloni, B. Salvant, and C. Tambasco, Instability latency in the LHC, in Proceedings of the 10th International Particle Accelerator Conference, Melbourne, Australia, May 2019, pp. 3204-3207, https:// doi.org/10.18429/JACoW-IPAC2019-WEPTS044.

[7] S. V. Furuseth, X. Buffat, E. Métral, D. Valuch, B. Salvant, D. Amorim, N. Mounet, M. E. Söderén, S. A. Antipov, T. Pieloni, and C. Tambasco, MD3288: Instability latency with controlled noise, CERN, Geneva, Switzerland, Report No. CERN-ACC-NOTE-2019-0011, 2019.

[8] X. Buffat, Transverse beams stability studies at the Large Hadron Collider, Ph.D. thesis, École polytechnique fédérale de Lausanne, Lausanne, Switzerland, 2015, Report No. CERN-THESIS-2014-246.

[9] S. V. Furuseth and X. Buffat, Long-term evolution of Landau damping in the presence of transverse noise, feedback, and detuning, Phys. Rev. Accel. Beams 23, 034401 (2020).

[10] Goldstein, Safko, and Poole, Classical Mechanics, 3rd ed. (Pearson Education, Harlow, UK, 2014).

[11] A. A. Vlasov, On the kinetic theory of an assembly of particles with collective interaction, Russ. Phys. J. 9, 25 (1945).

[12] G. Besnier, Contribution à La Théorie de La Stabilité Des Oscillations Longitudinales d'un Faisceau Accéléré En Régime de Charge d'espace, Ph.D. thesis, Université de Rennes, Rennes, France, 1978.

[13] J. S. Berg, Coherent modes for multiple non-rigid bunches in a storage ring, Ph.D. thesis, Stanford University, Stanford, CA, 1996, SLAC-REPORT-478.

[14] N. Mounet, Vlasov solvers and macroparticle simulations, CERN Yellow Reports 1, 77 (2018); Report No. CERN2018-003-CP, https://doi.org/10.23732/CYRCP-2018001.77 .

[15] N. Mounet, Direct Vlasov solvers, in Proc. CAS-CERN Accelerator School on Numerical Methods for Analysis, Design and Modelling of Particle Accelerators, Thessaloniki, Greece, Nov. 2018, pp. 273-304, Report No. CERNACC-2020-0012.

[16] Y.H. Chin, Hamiltonian formulation for transverse bunched beam instabilities in the presence of Betatron tune spread, CERN, Geneva, Switzerland, Report No. CERN-SPS-85-9-DI-MST.

[17] A. Chao, Physics of Collective Beam Instabilities in High Energy Accelerators (John Wiley \& Sons, New York, 1994).

[18] A. Wolski, Beam Dynamics in High Energy Particle Accelerators (Imperial College Press, London, 2014). 
[19] J. Gareyte, J. P. Koutchouk, and F. Ruggiero, Landau damping, dynamic aperture and octupoles in LHC, CERN, Geneva, Switzerland, Report No. CERN-LHC-PROJECTREPORT-091, 1997.

[20] K. Y. Ng, Physics of Intensity Dependent Beam Instabilities (World Scientific, Singapore, 2006).

[21] J. L. Laclare, Introduction to coherent instabilitiesCoasting beam case, CERN, Geneva, Switzerland, Report No. CERN-85-19-V-2, 1985.

[22] N. G. Van Kampen, On the theory of stationary waves in plasmas, Physica 21, 949 (1955).

[23] Y. Chin and K. Yokoya, Nonlinear perturbation approach to bunch lengthening and blow-up of energy spread, Nucl. Instrum. Methods Phys. Res. 226, 223 (1984).

[24] M. Morrone, M. Martino, R. De Maria, M. Fitterer, and C. Garion, Magnetic frequency response of High-Luminosity Large Hadron Collider beam screens, Phys. Rev. Accel. Beams 22, 013501 (2019).

[25] X. Buffat, W. Herr, T. Pieloni, and D. Valuch, Modeling of the emittance growth due to decoherence in collision at the Large Hadron Collider, Phys. Rev. Accel. Beams 23, 021002 (2020).

[26] P. Baudrenghien and T. Mastoridis, Transverse emittance growth due to rf noise in the high-luminosity LHC crab cavities, Phys. Rev. Accel. Beams 18, 101001 (2015).

[27] A. Bazzani, S. Siboni, and G. Turchetti, Diffusion in Hamiltonian systems with a small stochastic perturbation, Physica D (Amsterdam) 76, 8 (1994).

[28] A. Bazzani and L. Beccaceci, Diffusion in Hamiltonian systems driven by harmonic noise, J. Phys. A 31, 5843 (1998).

[29] W. Herr, Introduction to Landau damping, in Proceedings of the CAS-CERN Accelerator School on Intensity Limitations in Particle Beams (CERN, Geneva, 2017), Vol. 3.

[30] X. Buffat, W. Herr, N. Mounet, T. Pieloni, and S. White, Stability diagrams of colliding beams in the Large Hadron Collider, Phys. Rev. Accel. Beams 17, 111002 (2014).
[31] S. V. Furuseth and X. Buffat, Parallel high-performance multi-beam multi-bunch simulations, Comput. Phys. Commun. 244, 180 (2019).

[32] Harpy, http://pylhc.github.io/Beta-Beat.src/harmonic_ analysis/, accessed 2019-10-28.

[33] G. Trad, A. Alexopoulos, E. Bravin, D. Butti, R. Kieffer, F. Roncarolo, S. Vlachos, and B. Wurkner, LHC emittance measurements in run II, in Proceedings of the 9th Evian Workshop on LHC Beam Operation, Evian-les-Bains, France, Rep. CERN-ACC-2019-059, 2019.

[34] S. Papadopoulou, F. Antoniou, I. Efthymiopoulos, M. Hostettler, G. Iadarola, N. Karastathis, S. Kostoglou, Y. Papaphilippou, and G. Trad, Monitoring and modelling of the LHC emittance and luminosity evolution in 2018, J. Phys. Conf. Ser. 1350, 012011 (2019).

[35] L. R. Carver et al., Instabilities and beam induced heating in 2015, in Proc. of the 6th Evian Workshop on LHC Beam Operation, Evian-les-Bains, France, Dec. 2015 (CERN, Geneva, Switzerland, 2015), pp. 95-100.

[36] R. Bruce, N. Fuster-Martínez, A. Mereghetti, D. Mirarchi, and S. Redaelli, Review of LHC run 2 machine configurations, in Proceedings of the 9th Evian Workshop on LHC Beam Operation, Evian-les-Bains, France, Report No. CERN-ACC-2019-059, 2019.

[37] S. V. Furuseth and X. Buffat, Noise and possible loss of Landau damping-Noise excited Wakefields, in Proceedings of ICFA mini workshop on Mitigation of Coherent Beam Instabilities in particle accelerators, Zermatt, Switzerland, 2019 (to be published), https://indico.cern.ch/event/775147/ contributions/3366452/.

[38] R. Tomás, X. Buffat, S. White, J. Barranco, P. Gonçalves Jorge, and T. Pieloni, Beam-beam amplitude detuning with forced oscillations, Phys. Rev. Accel. Beams 20, 101002 (2017).

[39] S. Peggs and C. Tang, Nonlinear diagnostics using an AC dipole, Report No. RHIC/AP/159, 1998. https://www .agsrhichome.bnl.gov/AP/ap_notes/RHIC_AP_159.pdf. 